\title{
THE MINIMUM-UNCERTAINTY SQUEEZED STATES FOR ATOMS AND PHOTONS IN A CAVITY
}

\author{
SERGEY I. KRYUCHKOV, SERGEI K. SUSLOV, AND JOSÉ M. VEGA-GUZMÁN
}

\begin{abstract}
We describe a multi-parameter family of the minimum-uncertainty squeezed states for the harmonic oscillator in nonrelativistic quantum mechanics. They are derived by the action of corresponding maximal kinematical invariance group on the standard ground state solution. We show that the product of the variances attains the required minimum value $1 / 4$ only at the instances that one variance is a minimum and the other is a maximum, when the squeezing of one of the variances occurs. The generalized coherent states are explicitly constructed and their Wigner function is studied. The overlap coefficients between the squeezed, or generalized harmonic, and the Fock states are explicitly evaluated in terms of hypergeometric functions and the corresponding photon statistics are discussed. Some applications to quantum optics, cavity quantum electrodynamics, and superfocusing in channeling scattering are mentioned. Explicit solutions of the Heisenberg equations for radiation field operators with squeezing are found.
\end{abstract}

\section{An Introduction}

From the very beginning, nonclassical states of the linear Planck oscillator, in particular the coherent and squeezed states, have been a subject of considerable interest in quantum physics (see [34], [42], [62], [86], [90], [143], [144] and the references therein). They occur naturally on an atomic scale [14], [84] and, possibly, can be observed among vibrational modes of crystals and molecules [30], [31], [44], [52]. A single monochromatic mode of light also represents a harmonic oscillator system for which nonclassical states can be generated very efficiently by using the interaction of laser light with nonlinear optical media [17], [102], [109], [110], [111], [112], [131], [140], [167]. Generation of squeezed light with a single atom has been experimentally demonstrated [127]. On a macroscopic scale, the squeezed states are utilized for detection of gravitational waves [75] below the so-called vacuum noise level and without violation of the uncertainty relation [1], [46], [130], [162].

The past decades progress in generation of pure quantum states of motion of trapped particles provides not only a clear illustration of basic principles of quantum mechanics, but it also manifests the ultimate control of particle motion. These states are of interest from the standpoint of quantum measurement concepts and facilitate other applications including quantum computation (see [13], [19], [22], [24], [61], [68], [73], [84], [101], [117], [118], [121], [128], [135], [138] and the references therein).

Date: March 25, 2013.

1991 Mathematics Subject Classification. Primary 81Q05, 35C05. Secondary 42A38.

Key words and phrases. Time-dependent Schrödinger equation, generalized harmonic oscillators, Schrödinger group, dynamic invariants, coherent and squeezed states, minimum-uncertainty squeezed states, Wigner and Moyal functions, quantum optics, Jaynes-Cummings model, cavity QED, second quantization, radiation field oscillators, Heisenberg equations of motion. 
It is well known that the harmonic quantum states can be analyzed through the dynamics of a single, two-level atom which radiatively couples to the single mode radiation field in the JaynesCummings(-Paul) model [21], [25], [82], [101], [141], [147], [165] extensively studied in the cavity QED [45], [68], [132], [133]. Creation and detection of thermal, Fock, coherent, and squeezed states of motion of a single ${ }^{9} B e^{+}$ion confined in a rf Paul trap was reported in [117], where the state of atomic motion had been observed through the evolution of the atom's internal levels (e.g., collapse and revival) under the influence of a Jaynes-Cummings interaction realized with the application of external (classical) fields. The distribution over the Fock states is deduced from an analysis of Rabi oscillations.

Moreover, Fock, coherent, and squeezed states of motion of a harmonically bound cold cesium atoms were experimentally observed in a $1 D$ optical lattice [13], [121]. This method gives a direct access to the momentum distribution through the square of the modulus of the wavefunction in velocity space (see also [20], [21], [23], [26], [32], [73], [83], [84], [101], [128], [163] and the references therein regarding cold trapped ions and their nonclassical states; progress in atomic physics and quantum optics using superconducting circuits is reviewed in [59], [173]).

Recent reports on observations of the dynamical Casimir effect [95], [172] strengthen the interest to the nonclassical states of generalized harmonic oscillators [34], [35], [39], [40], [41], [67], [114], [115], [123], [157] and [166]. The amplification of quantum fluctuations by modulating parameters of an oscillator is closely related to the process of particle production in quantum fields [35], [80], [115], and [123]. Other dynamical amplification mechanisms include the Unruh effect [161] and Hawking radiation [12], [71], [72].

The purpose of this paper is to construct the minimum-uncertainty squeezed states for quantum harmonic oscillators, which are important in these applications, in the most simple closed form. Our approach reveals the quantum numbers/integrals of motion of the squeezed states in terms of solution of certain Ermakov-type system [104], [105]. The corresponding generalizations of Fock states, which were originally found in [116] and recently rediscovered in [105], are discussed in detail. As a result, the probability amplitudes of these nonclassical states of motion are explicitly evaluated in terms of hypergeometric functions. Their experimental observations in cavity QED and quantum optics are briefly reviewed. Moreover, the radiation field operators of squeezed photons, which can be created from the QED vacuum, are introduced by second quantization with the aid of hidden symmetry of harmonic oscillator problem in the Heisenberg picture.

In summary, experimental recognitions of the nonclassical harmonic states of motion have been achieved through reconstruction of the Wigner function in optical quantum-state tomography [17], [112], from a Fourier analysis of Rabi oscillations of a trapped atom [117], and/or by a direct observation of the square of the modulus of the wavefunction for a large sample of cold cesium atoms in a $1 D$ optical lattice [13], [121]. Our theoretical consideration complements all of these advanced experimental techniques by identifying the state quantum numbers from first principles. This approach may provide a guidance for engineering more advanced nonclassical states.

The paper is organized as follows. In sections 2 and 3, we describe the minimum-uncertainty squeezed states for the linear harmonic oscillator in coordinate representation. The generalized coherent, or TCS states, are constructed in section 4. In sections 4 and 5, the Wigner and Moyal functions of the squeezed states are evaluated directly from the corresponding wavefunctions and their classical time evolution is verified with the help of a computer algebra system. The eigenfunction expansions of the squeezed (or generalized harmonic) states in terms of the standard Fock 
ones are derived in section 6 (see also [38], [43], [87] and the references therein for important special cases). Some experiments on engineering of nonclassical states of motion are analyzed in section 7 . Here, the experimentally observed probability distributions are derived from our explicit expression for the probability amplitudes obtained in the previous section. Theoretically predicted in [30], [31], superfocusing in channel scattering is also discussed. In section 8, we revisit the radiation field quantization in a perfect cavity, which is important for applications to quantum optics. Nonstandard solutions of the Heisenberg equations of motion for the electromagnetic field operators, that naturally describe squeezing in the Heisenberg picture, are found. The variance of the number operator, which together with the eigenfunction expansion allows one to compare our results with experimentally observed squeezed photon statistics [17], [140], is evaluated from first principles in section 9. A brief summary is provided in the last section. A compact complex parametrization of the Schrödinger group can be found in appendix.

\section{The Minimum-Uncertainty Squeezed States}

The Heisenberg Uncertainty Principle is one of the fundamental laws of nature and the coherent states that minimize this uncertainty relation are well known. But, equally important in recent developments, minimum-uncertainty squeezed states are not so familiar outside a relatively narrow group of experts. Here, for the benefits of the reader, we construct these states as explicitly as possible and elaborate on some of their remarkable features.

The time-dependent Schrödinger equation for the simple harmonic oscillator in one dimension,

$$
2 i \psi_{t}+\psi_{x x}-x^{2} \psi=0,
$$

has the following square integrable solution (Gaussian wave packet):

$$
\psi_{0}(x, t)=e^{i\left(\alpha(t) x^{2}+\delta(t) x+\kappa(t)+\gamma(t)\right)} \sqrt{\frac{\beta(t)}{\sqrt{\pi}}} e^{-(\beta(t) x+\varepsilon(t))^{2} / 2},
$$

where

$$
\begin{aligned}
& \alpha(t)=\frac{\alpha_{0} \cos 2 t+\sin 2 t\left(\beta_{0}^{4}+4 \alpha_{0}^{2}-1\right) / 4}{\beta_{0}^{4} \sin ^{2} t+\left(2 \alpha_{0} \sin t+\cos t\right)^{2}}, \\
& \beta(t)=\frac{\beta_{0}}{\sqrt{\beta_{0}^{4} \sin ^{2} t+\left(2 \alpha_{0} \sin t+\cos t\right)^{2}}}, \\
& \gamma(t)=\gamma_{0}-\frac{1}{2} \arctan \frac{\beta_{0}^{2} \tan t}{1+2 \alpha_{0} \tan t}, \\
& \delta(t)=\frac{\delta_{0}\left(2 \alpha_{0} \sin t+\cos t\right)+\varepsilon_{0} \beta_{0}^{3} \sin t}{\beta_{0}^{4} \sin ^{2} t+\left(2 \alpha_{0} \sin t+\cos t\right)^{2}}, \\
& \varepsilon(t)=\frac{\varepsilon_{0}\left(2 \alpha_{0} \sin t+\cos t\right)-\beta_{0} \delta_{0} \sin t}{\sqrt{\beta_{0}^{4} \sin ^{2} t+\left(2 \alpha_{0} \sin t+\cos t\right)^{2}}}, \\
& \kappa(t)=\kappa_{0}+\sin ^{2} t \frac{\varepsilon_{0} \beta_{0}^{2}\left(\alpha_{0} \varepsilon_{0}-\beta_{0} \delta_{0}\right)-\alpha_{0} \delta_{0}^{2}}{\beta_{0}^{4} \sin ^{2} t+\left(2 \alpha_{0} \sin t+\cos t\right)^{2}}
\end{aligned}
$$




$$
+\frac{1}{4} \sin 2 t \frac{\varepsilon_{0}^{2} \beta_{0}^{2}-\delta_{0}^{2}}{\beta_{0}^{4} \sin ^{2} t+\left(2 \alpha_{0} \sin t+\cos t\right)^{2}}
$$

$\left(\alpha_{0}, \beta_{0} \neq 0, \gamma_{0}, \delta_{0}, \varepsilon_{0}, \kappa_{0}\right.$ are real-valued initial data of the corresponding Ermakov-type system; a complex form of equations (2.3)-(2.8) is provided in Appendix A and the invariants are given by (6.11)-(6.12); in what follows one may choose $\left.\gamma_{0}=\kappa_{0}=0\right)$. This quantum state can be thought of as a special case of a 'nonclassical' oscillator solution originally found by Marhic [116]. The latter has been recently derived in a unified approach to generalized harmonic oscillators (see, for example, [27], [29], [98], [105], [153] and the references therein). These solutions can be verified by a direct substitution with the aid of Mathematica computer algebra system [91] (see also [92]), [105], and [106]. (In retrospect, the simplest special case $\beta_{0}= \pm 1$ and $\alpha_{0}=\gamma_{0}=\delta_{0}=\varepsilon_{0}=\kappa_{0}=0$ is the ground oscillator state [55], [64], [96], [119], [143], [144]. For the coherent states [144], $\alpha_{0}=0$ and $\beta_{0}= \pm 1$, while a more general wave packet with $\alpha_{0}=0$ was discussed in [76], [77]. Derivation of these formulas can be found in Refs. [104], [105], and [116]. An analog of Berry's phase is evaluated in Refs. [158], [159].)

The "dynamic harmonic oscillator ground state" (2.2)-(2.8) is the eigenfunction,

$$
E(t) \psi_{0}(x, t)=\frac{1}{2} \psi_{0}(x, t)
$$

of the time-dependent dynamical invariant,

$$
\begin{aligned}
E(t) & =\frac{1}{2}\left[\frac{(p-2 \alpha x-\delta)^{2}}{\beta^{2}}+(\beta x+\varepsilon)^{2}\right] \\
& =\frac{1}{2}\left[\widehat{a}(t) \widehat{a}^{\dagger}(t)+\widehat{a}^{\dagger}(t) \widehat{a}(t)\right], \quad \frac{d}{d t}\langle E\rangle=0,
\end{aligned}
$$

with a familiar operator identity:

$$
\frac{\partial E}{\partial t}+i^{-1}[E, H]=0, \quad H=\frac{1}{2}\left(p^{2}+x^{2}\right) .
$$

The time-dependent annihilation $\widehat{a}(t)$ and creation $\widehat{a}^{\dagger}(t)$ operators are given by the following Bogoliubov-type transformation:

$$
\begin{aligned}
& \widehat{a}(t)=\frac{1}{\sqrt{2}}\left(\beta x+\varepsilon+i \frac{p-2 \alpha x-\delta}{\beta}\right), \\
& \widehat{a}^{\dagger}(t)=\frac{1}{\sqrt{2}}\left(\beta x+\varepsilon-i \frac{p-2 \alpha x-\delta}{\beta}\right)
\end{aligned}
$$

where $p=i^{-1} \partial / \partial x$, in terms of solutions (2.3)-(2.8) [105]. They satisfy the canonical commutation relation,

$$
\widehat{a}(t) \widehat{a}^{\dagger}(t)-\widehat{a}^{\dagger}(t) \widehat{a}(t)=1,
$$

and the spectrum of invariant $E$ can be obtained by using the Heisenberg-Weyl algebra (a "second quantization", the Fock states [57], [58], [129]). In particular,

$$
\widehat{a}(t) \Psi_{0}(x, t)=0, \quad \psi_{0}(x, t)=e^{i \gamma(t)} \Psi_{0}(x, t),
$$

for the corresponding "vacuum state".

This form of quadratic dynamical invariant and creation and annihilation operators for the generalized harmonic oscillators have been obtained in [137] (see also [29], [40], [41], [156] and the 
references therein for important special cases). An application to the electromagnetic-field quantization is discussed in [93] (see also section 8).

The maximum kinematical invariance groups of the free particle and harmonic oscillator were introduced in [5], [6], [66], [79], [124], and [125] (see also [16], [85], [120], [134], [154], [155] and the references therein). We use connections with the Ermakov-type system [104], [105] (see [49], [99] and the references therein regarding the Ermakov equation). A general procedure of obtaining new solutions by using enveloping algebra of generators of the Heisenberg-Weyl group is described in [41] (see also [7], [9], [40], [60], [116] regarding the corresponding wavefunctions).

\section{The Uncertainty Relation and Squeezing}

A quantum state is said to be "squeezed" if its oscillating variances $\left\langle(\Delta p)^{2}\right\rangle$ and $\left\langle(\Delta x)^{2}\right\rangle$ become smaller than the variances of the "static" vacuum state $\left\langle(\Delta p)^{2}\right\rangle=\left\langle(\Delta x)^{2}\right\rangle=1 / 2($ with $\hbar=1)$. For the harmonic oscillator, the product of the variances attains a minimum value only at the instances when one variance is a minimum and the other is a maximum. If the minimum value of the product is equal to $1 / 4$, then the state is called a minimum-uncertainty squeezed state (see, for example, [45], [70], [87], [146], [151], [152], [168], and [174]). This property can be easily verified for solution $(2.2)$.

According to the transform (2.12), the corresponding expectation values oscillate sinusoidally in time

$$
\begin{array}{ll}
\langle x\rangle=-\frac{1}{\beta_{0}}\left[\left(2 \alpha_{0} \varepsilon_{0}-\beta_{0} \delta_{0}\right) \sin t+\varepsilon_{0} \cos t\right], & \frac{d}{d t}\langle x\rangle=\langle p\rangle, \\
\langle p\rangle=-\frac{1}{\beta_{0}}\left[\left(2 \alpha_{0} \varepsilon_{0}-\beta_{0} \delta_{0}\right) \cos t-\varepsilon_{0} \sin t\right], & \frac{d}{d t}\langle p\rangle=-\langle x\rangle
\end{array}
$$

with the initial data $\left.\langle x\rangle\right|_{t=0}=-\varepsilon_{0} / \beta_{0}$ and $\left.\langle p\rangle\right|_{t=0}=-\left(2 \alpha_{0} \varepsilon_{0}-\beta_{0} \delta_{0}\right) / \beta_{0}$. This provides a connection of these parameters with the Ehrenfest theorem [47], [69], [170].

The expectation values $\langle x\rangle$ and $\langle p\rangle$ satisfy the classical equation for harmonic motion, $y^{\prime \prime}+y=0$, with the total "classical mechanical energy" given by

$$
\frac{1}{2}\left[\langle p\rangle^{2}+\langle x\rangle^{2}\right]=\frac{\left(2 \alpha_{0} \varepsilon_{0}-\beta_{0} \delta_{0}\right)^{2}+\varepsilon_{0}^{2}}{2 \beta_{0}^{2}}=\left.\frac{1}{2}\left[\langle p\rangle^{2}+\langle x\rangle^{2}\right]\right|_{t=0} .
$$

For the standard deviations on solution (2.2)-(2.8), one gets

$$
\begin{aligned}
& \left\langle(\Delta p)^{2}\right\rangle=\left\langle p^{2}\right\rangle-\langle p\rangle^{2} \\
& =\frac{1+4 \alpha_{0}^{2}+\beta_{0}^{4}+\left(4 \alpha_{0}^{2}+\beta_{0}^{4}-1\right) \cos 2 t-4 \alpha_{0} \sin 2 t}{4 \beta_{0}^{2}}, \\
& \left\langle(\Delta x)^{2}\right\rangle=\left\langle x^{2}\right\rangle-\langle x\rangle^{2} \\
& =\frac{1+4 \alpha_{0}^{2}+\beta_{0}^{4}-\left(4 \alpha_{0}^{2}+\beta_{0}^{4}-1\right) \cos 2 t+4 \alpha_{0} \sin 2 t}{4 \beta_{0}^{2}},
\end{aligned}
$$

and

$$
\left\langle(\Delta p)^{2}\right\rangle\left\langle(\Delta x)^{2}\right\rangle=\frac{1}{16 \beta_{0}^{4}}
$$




$$
\times\left[\left(1+4 \alpha_{0}^{2}+\beta_{0}^{4}\right)^{2}-\left(\left(4 \alpha_{0}^{2}+\beta_{0}^{4}-1\right) \cos 2 t-4 \alpha_{0} \sin 2 t\right)^{2}\right] .
$$

Here,

$$
\begin{gathered}
\sigma_{p}=\left\langle(\Delta p)^{2}\right\rangle=\frac{4 \alpha^{2}+\beta^{4}}{2 \beta^{2}}, \quad \sigma_{x}=\left\langle(\Delta x)^{2}\right\rangle=\frac{1}{2 \beta^{2}} \\
\sigma_{p x}=\frac{1}{2}\langle\Delta p \Delta x+\Delta x \Delta p\rangle=\frac{\alpha}{\beta^{2}}
\end{gathered}
$$

with two invariants:

$$
\sigma_{p}+\sigma_{x}=\frac{4 \alpha^{2}+\beta^{4}+1}{2 \beta^{2}}=\frac{4 \alpha_{0}^{2}+\beta_{0}^{4}+1}{2 \beta_{0}^{2}}, \quad\left|\begin{array}{cc}
\sigma_{p} & \sigma_{p x} \\
\sigma_{p x} & \sigma_{x}
\end{array}\right|=\sigma_{p} \sigma_{x}-\sigma_{p x}^{2}=\frac{1}{4}
$$

(More invariants are given by in (6.11)-(6.12).) The Schrödinger minimum-uncertainty states [144], when $\left\langle(\Delta p)^{2}\right\rangle=\left\langle(\Delta x)^{2}\right\rangle=1 / 2$, are defined by taking $\alpha_{0}=0$ and $\beta_{0}^{2}=1$. For the ground state solution, when $\alpha_{0}=\delta_{0}=\varepsilon_{0}=0$ and $\beta_{0}= \pm 1$, one gets $\langle x\rangle=\langle p\rangle \equiv 0$ and

$$
\left\langle(\Delta p)^{2}\right\rangle=\left\langle(\Delta x)^{2}\right\rangle=\frac{1}{2}
$$

as presented in the textbooks [55], [64], [65], [70], [96], [119], [129].

By adding (3.3)-(3.5), we arrive at

$$
\begin{aligned}
& \langle H\rangle=\frac{1}{2}\left[\left\langle p^{2}\right\rangle+\left\langle x^{2}\right\rangle\right] \\
& =\frac{1+4 \alpha_{0}^{2}+\beta_{0}^{4}}{4 \beta_{0}^{2}}+\frac{\left(2 \alpha_{0} \varepsilon_{0}-\beta_{0} \delta_{0}\right)^{2}+\varepsilon_{0}^{2}}{2 \beta_{0}^{2}} \geq \frac{1}{2}
\end{aligned}
$$

for the total "quantum mechanical energy" in terms of integrals of motion (the vacuum value $1 / 2$ occurs when $\beta_{0}= \pm 1$ and $\left.\alpha_{0}=\delta_{0}=\varepsilon_{0}=0\right)$. See also [17] and [43].

Therefore, the upper and lower bound in the Heisenberg uncertainty relation are given by

$$
\max \left[\left\langle(\Delta p)^{2}\right\rangle\left\langle(\Delta x)^{2}\right\rangle\right]=\frac{\left(1+4 \alpha_{0}^{2}+\beta_{0}^{4}\right)^{2}}{16 \beta_{0}^{4}}, \quad \text { when } \quad \cot 2 t=\frac{4 \alpha_{0}}{4 \alpha_{0}^{2}+\beta_{0}^{4}-1}
$$

and

$$
\min \left[\left\langle(\Delta p)^{2}\right\rangle\left\langle(\Delta x)^{2}\right\rangle\right]=\frac{1}{4}, \quad \text { if } \quad \tan 2 t=-\frac{4 \alpha_{0}}{4 \alpha_{0}^{2}+\beta_{0}^{4}-1} .
$$

Our explicit formulas (3.4)-(3.5) show that the product of the variances attains the minimum value $1 / 4$ only at the instances that one variance is a minimum and the other is a maximum as stated in [70]. Here, squeezing of one of the variances is explicitly described. Indeed,

$$
\left(4 \alpha_{0}^{2}+\beta_{0}^{4}-1\right) \cos 2 t-4 \alpha_{0} \sin 2 t= \pm\left(4 \alpha_{0}^{2}+\left(\beta_{0}^{2}+1\right)^{2}\right)^{1 / 2}\left(4 \alpha_{0}^{2}+\left(\beta_{0}^{2}-1\right)^{2}\right)^{1 / 2}
$$

under the minimization condition (3.12) and at the minimum

$$
\begin{aligned}
& \left\langle(\Delta p)^{2}\right\rangle=\frac{1}{4 \beta_{0}^{2}}\left[1+4 \alpha_{0}^{2}+\beta_{0}^{4} \pm\left(4 \alpha_{0}^{2}+\left(\beta_{0}^{2}+1\right)^{2}\right)^{1 / 2}\left(4 \alpha_{0}^{2}+\left(\beta_{0}^{2}-1\right)^{2}\right)^{1 / 2}\right] \\
& \left\langle(\Delta x)^{2}\right\rangle=\frac{1}{4 \beta_{0}^{2}}\left[1+4 \alpha_{0}^{2}+\beta_{0}^{4} \mp\left(4 \alpha_{0}^{2}+\left(\beta_{0}^{2}+1\right)^{2}\right)^{1 / 2}\left(4 \alpha_{0}^{2}+\left(\beta_{0}^{2}-1\right)^{2}\right)^{1 / 2}\right]
\end{aligned}
$$


for all real values of our parameters. At this instant the squeezing occur:

$$
\left\langle(\Delta p)^{2}\right\rangle>\frac{1}{2}\left(<\frac{1}{2}\right), \quad\left\langle(\Delta x)^{2}\right\rangle<\frac{1}{2}\left(>\frac{1}{2}\right)
$$

(for upper and lower signs, respectively). As a result, the minimum-uncertainty squeezed states for the linear harmonic oscillator are presented in closed form (2.3)-(2.8) (see also [70] for numerical simulations). A natural generalization will be discussed in the next section. The corresponding wavefunction in the momentum representation can be derived by the (inverse) Fourier transform of (2.2) and (2.3)-(2.8) (see [105] for more details). Experimentally observed time-oscillations of the velocity variance [121] reveal certain damping, which can be explain in models of quantum damped oscillators discussed in [28], [29], and [40] (see also the references therein).

\section{An Extension: the TCS States}

We construct an analog of the coherent states (generalized coherent, or the TCS states in the terminology of Ref. [174]) in a standard fashion

$$
\begin{aligned}
& \psi(x, t)=e^{-|\zeta|^{2} / 2} \sum_{n=0}^{\infty} \psi_{n}(x, t) \frac{\zeta^{n}}{\sqrt{n !}} \\
& =e^{-|\eta|^{2} / 2} e^{i \gamma} \sum_{n=0}^{\infty} \Psi_{n}(x, t) \frac{\eta^{n}}{\sqrt{n !}}, \quad \eta=\zeta e^{2 i \gamma},
\end{aligned}
$$

where $\zeta$ is an arbitrary complex parameter and the "dynamic" wavefunctions are given by equations (1.2) and (1.16) of [105] reproduced here for the reader's convenience:

$$
\psi_{n}(x, t)=e^{i\left(\alpha x^{2}+\delta x+\kappa\right)+i(2 n+1) \gamma} \sqrt{\frac{\beta}{2^{n} n ! \sqrt{\pi}}} e^{-\xi^{2} / 2} H_{n}(\xi), \quad \xi=\beta x+\varepsilon
$$

(see also [40] and [116]), where $H_{n}(x)$ are the Hermite polynomials [126]. In the explicit form [144],

$$
\begin{aligned}
\psi(x, t) & =\sqrt{\frac{\beta}{\sqrt{\pi}}} e^{-\left(\xi^{2}+|\eta|^{2}\right) / 2} e^{i\left(\alpha x^{2}+\delta x+\kappa+\gamma\right)} \sum_{n=0}^{\infty}\left(\frac{\eta}{\sqrt{2}}\right)^{n} \frac{H_{n}(\xi)}{n !} \\
& =\sqrt{\frac{\beta}{\sqrt{\pi}}} e^{\left(\eta^{2}-|\eta|^{2}\right) / 2} e^{i\left(\alpha x^{2}+\delta x+\kappa+\gamma\right)} e^{-(\xi-\sqrt{2} \eta)^{2} / 2},
\end{aligned}
$$

and the eigenvalue problem is given by [174]:

$$
\widehat{a}(t) \psi(x, t)=\eta \psi(x, t) .
$$

An elementary calculation shows that on these "dynamic coherent states",

$$
\langle x\rangle=\frac{1}{\beta \sqrt{2}}\left(\eta+\eta^{*}\right)-\frac{\varepsilon}{\beta},\left.\quad\langle x\rangle\right|_{t=0}=\frac{\sqrt{2}}{\beta_{0}}|\zeta| \cos \left(2\left(\gamma_{0}+\phi\right)\right)-\frac{\varepsilon_{0}}{\beta_{0}},
$$

and

$$
\langle p\rangle=\frac{\beta}{i \sqrt{2}}\left(\eta-\eta^{*}\right)+\frac{\alpha \sqrt{2}}{\beta}\left(\eta+\eta^{*}\right)+\left(\delta-\frac{2 \alpha \varepsilon}{\beta}\right),
$$




$$
\left.\langle p\rangle\right|_{t=0}=\beta_{0} \sqrt{2}|\zeta| \sin \left(2\left(\gamma_{0}+\phi\right)\right)+2^{3 / 2} \frac{\alpha_{0}}{\beta_{0}}|\zeta| \cos \left(2\left(\gamma_{0}+\phi\right)\right)+\left(\delta_{0}-\frac{2 \alpha_{0} \varepsilon_{0}}{\beta_{0}}\right),
$$

if $\zeta=|\zeta| e^{2 i \phi}$. Moreover, a direct Mathematica verification shows that these expectation values satisfy the required classical equation for simple harmonic motion.

A similar calculation reveals that the corresponding oscillating variances $\left\langle(\Delta p)^{2}\right\rangle$ and $\left\langle(\Delta x)^{2}\right\rangle$ coincide with those for the "dynamic vacuum states" given by (3.4)-(3.5). The "dynamic coherent states" (4.3) are also the minimum-uncertainty squeezed states but they are not eigenfunctions of the time-dependent dynamic invariant (2.10) when $\eta \neq 0$.

The Wigner function [74], [102], [141], [142], [171],

$$
W(x, p)=\frac{1}{2 \pi} \int_{-\infty}^{\infty} \psi^{*}(x+y / 2) \psi(x-y / 2) e^{i p y} d y
$$

for the TCS states (4.3) is given by

$$
W(x, p)=\frac{1}{\pi} \exp \left[-\left(P+i \frac{\eta-\eta^{*}}{\sqrt{2}}\right)^{2}-\left(Q-\frac{\eta+\eta^{*}}{\sqrt{2}}\right)^{2}\right]
$$

where

$$
P=\frac{p-2 \alpha x-\delta}{\beta}, \quad Q=\beta x+\varepsilon .
$$

In view of (4.5)-(4.6), we arrive at the following expression of the Wigner function:

$$
W(x, p)=\frac{1}{\pi} \exp \left[-\frac{(p-\langle p\rangle)^{2}}{\beta^{2}}+\frac{4 \alpha}{\beta^{2}}(p-\langle p\rangle)(x-\langle x\rangle)-\frac{4 \alpha^{2}+\beta^{4}}{\beta^{2}}(x-\langle x\rangle)^{2}\right],
$$

in terms of the classical trajectories $\langle x\rangle$ and $\langle p\rangle$ and the solutions of Ermakov-type system (2.3)(2.4). Taking into account the time-dependent variances (3.7), one gets [38], [43], [142], [150]:

$$
W(x, p)=\frac{1}{\pi} \exp \left[-2\left(\sigma_{x}(p-\langle p\rangle)^{2}-2 \sigma_{p x}(p-\langle p\rangle)(x-\langle x\rangle)+\sigma_{p}(x-\langle x\rangle)^{2}\right)\right],
$$

where $\sigma_{p}, \sigma_{x}$, and $\sigma_{p x}$ are given by (3.7). Then

$$
W(x, p ; t)=W(x \cos t-p \sin t, x \sin t+p \cos t ; t=0)
$$

by a direct calculation - the graph of Wigner function rotates in the phase plane without changing its shape [150]. (In a traditional approach, the quantum Liouville equation of motion for Wigner function of the corresponding quadratic system is used in order to determine this time evolution [141]. We have obtained the same result directly from the wavefunctions; see also [142].) Some Mathematica animations can be found in Ref. [94]. Reconstruction of the original wavefunction from the Wigner quasidistribution is discussed in Refs. [100], [160]. (See also Ref. [88] for a detailed discussion of fundamental limitations on simultaneous range-velocity determination in radar systems with the aid of a quantum mechanical analog to the Wigner distribution function.) 


\section{The Moyal Functions}

The total energy of a "dynamic harmonic state" (4.2) can be presented as

$$
\langle H\rangle=\frac{1}{2}\left[\left\langle p^{2}\right\rangle+\left\langle x^{2}\right\rangle\right]=\left(n+\frac{1}{2}\right) \frac{1+4 \alpha_{0}^{2}+\beta_{0}^{4}}{2 \beta_{0}^{2}}+\frac{\left(2 \alpha_{0} \varepsilon_{0}-\beta_{0} \delta_{0}\right)^{2}+\varepsilon_{0}^{2}}{2 \beta_{0}^{2}}
$$

by (A.3)-(A.5) of Ref. [105].

The Moyal functions [122] for the "dynamic harmonic states" (4.2):

$$
W_{m n}(x, p, t)=\frac{1}{2 \pi} \int_{-\infty}^{\infty} \psi_{m}^{*}(x+y / 2, t) \psi_{n}(x-y / 2, t) e^{i p y} d y
$$

can be evaluated in terms of Laguerre and Charlier polynomials in the standard way [88], [126], [141], [142]:

$$
\begin{aligned}
W_{m n}(x, p, t)= & \frac{(-1)^{m} e^{2 i(n-m) \gamma}}{\pi} e^{-Q^{2}-P^{2}} 2^{(m-n) / 2} \sqrt{\frac{m !}{n !}} \\
& \times(Q-i P)^{n-m} L_{m}^{n-m}\left(2\left(Q^{2}+P^{2}\right)\right)
\end{aligned}
$$

in the notation (4.9). Once again, the time evolution of the corresponding Wigner function $W_{n n}(x, p, t)$ is defined by equation (4.12).

In the case of an arbitrary linear combination,

$$
\psi(x, t)=\sum_{m} c_{m} \psi_{m}(x, t)
$$

the Wigner function can be obtain as a double sum of Moyal's functions:

$$
W(x, p, t)=\sum_{m, n} c_{m}^{*} c_{n} W_{m n}(x, p, t) .
$$

A coherent superposition of two Fock states with $n=0$ and $n=1$ was experimentally realized in [121]. Moreover, the state of the electromagnetic field can be chosen anywhere between the single-photon and squeezed state in Ref. [81].

\section{Eigenfunction Expansions}

Experimentally observed statistics for various squeezed states of photons and ions in a box [17], [68], [101], [112], [117], [140] can be naturally explained in terms of explicit developments with respect to the Fock states. For a linear harmonic oscillator in coordinate representation, we consider the corresponding wavefunctions and use known expansions in Hermite polynomials [97], [103], [126]. Group-theoretical properties are discussed, for example, in Refs. [41], [87], [126], [129].

6.1. Familiar Expansions. For the stationary harmonic oscillator wavefunctions,

$$
\Psi_{n}(x)=\frac{e^{-x^{2} / 2}}{\sqrt{2^{n} n ! \sqrt{\pi}}} H_{n}(x)
$$

there are two well known expansions:

$$
e^{i(\Gamma+B x)} \Psi_{n}(x+A)=\sum_{m=0}^{\infty} T_{m n}(A, B, \Gamma) \Psi_{m}(x),
$$


where

$$
\begin{aligned}
T_{m n}(A, B, \Gamma)= & \int_{-\infty}^{\infty} \Psi_{m}^{*}(x) e^{i(\Gamma+B x)} \Psi_{n}(x+A) d x \\
= & \frac{i^{m-n}}{\sqrt{m ! n !}} e^{i(\Gamma-A B / 2)} e^{-\nu / 2}\left(\frac{i A+B}{\sqrt{2}}\right)^{m}\left(\frac{i A-B}{\sqrt{2}}\right)^{n} \\
& \times{ }_{2} F_{0}\left(-n,-m ;-\frac{1}{\nu}\right)
\end{aligned}
$$

with $\nu=\left(A^{2}+B^{2}\right) / 2$ (see, for example, [103], [126] for relations with the Heisenberg-Weyl group, Charlier polynomials, and Poisson distribution) and

$$
e^{i \alpha x^{2}} \Psi_{n}(\beta x)=\sum_{m=0}^{\infty} M_{m n}(\alpha, \beta) \Psi_{m}(x) .
$$

By the orthogonality,

$$
M_{m n}(\alpha, \beta)=\int_{-\infty}^{\infty} \Psi_{m}^{*}(x) e^{i \alpha x^{2}} \Psi_{n}(\beta x) d x
$$

and one can use the integral evaluated by Bailey:

$$
\begin{aligned}
& \int_{-\infty}^{\infty} e^{-\lambda^{2} x^{2}} H_{m}(a x) H_{n}(b x) d x \\
& =\frac{2^{m+n}}{\lambda^{m+n+1}} \Gamma\left(\frac{m+n+1}{2}\right)\left(a^{2}-\lambda^{2}\right)^{m / 2}\left(b^{2}-\lambda^{2}\right)^{n / 2}
\end{aligned}
$$

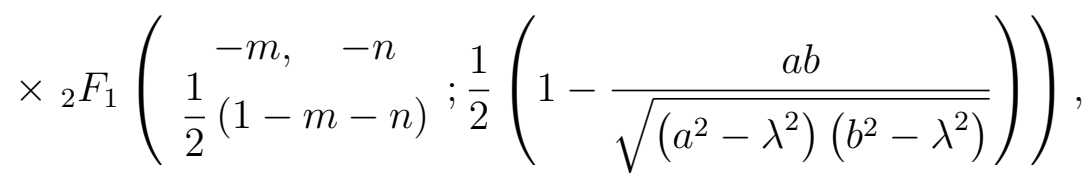

$\operatorname{Re} \lambda^{2}>0$, if $m+n$ is even; the integral vanishes by symmetry if $m+n$ is odd; see [8], [107] and the references therein for earlier works on these integrals, some of their special cases and extensions. As a result,

$$
\begin{aligned}
& M_{m n}(\alpha, \beta)=i^{n} \sqrt{\frac{2^{m+n}}{m ! n ! \pi}} \Gamma\left(\frac{m+n+1}{2}\right) \\
& \times \frac{\left(\frac{1-\beta^{2}}{2}+i \alpha\right)^{m / 2}\left(\frac{1-\beta^{2}}{2}-i \alpha\right)^{n / 2}}{\left(\frac{1+\beta^{2}}{2}-i \alpha\right)^{(m+n+1) / 2}} \\
& \times{ }_{2} F_{1}\left(\frac{1}{2}(1-m-n) ; \frac{1}{2}\left(1 \pm \frac{2 i \beta}{\sqrt{4 \alpha^{2}+\left(\beta^{2}-1\right)^{2}}}\right)\right) .
\end{aligned}
$$

The terminating hypergeometric function can be transformed as follows

$$
{ }_{2} F_{1}\left(\begin{array}{c}
-k,-n \\
\frac{1}{2}(1-k-n)
\end{array} ; \quad \frac{1}{2}(1+i \zeta)\right)
$$




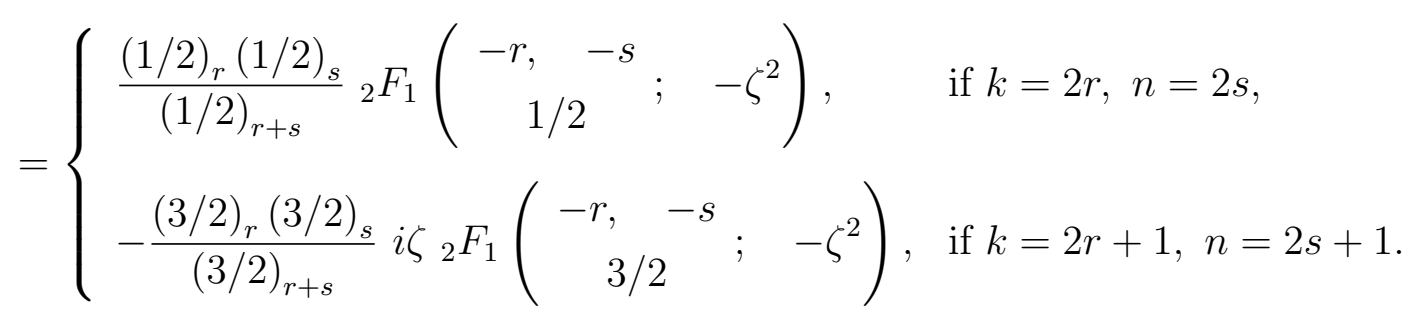

It is valid in the entire complex plane; the details are given in Appendix B of [97]. The latter transformation completes evaluation of the Bailey integral (6.6) and the matrix elements (6.7) in terms of the hypergeometric functions. (Relations with the group $S U(1,1)$, Meixner polynomials [126], and with two special cases of the negative binomial, or Pascal, distribution [97] are discussed elsewhere.)

6.2. Probability Amplitudes. Expansions (6.2) and (6.4) results in

$$
\psi_{n}(x, t)=e^{i(2 n+1)\left(\gamma-\gamma_{0}\right)} \sqrt{\beta} \sum_{m=0}^{\infty} C_{m n}(t) \Psi_{m}(x),
$$

where

$$
\begin{aligned}
C_{m n}(t) & =\sum_{k=0}^{\infty} M_{m k}(\alpha, \beta) T_{k n}\left(\varepsilon, \frac{\delta}{\beta}, \kappa\right) \\
& =\sum_{k=0}^{\infty} T_{m k}\left(\frac{\varepsilon}{\beta}, \delta-\frac{2 \alpha \varepsilon}{\beta}, \kappa-\frac{\alpha \varepsilon^{2}}{\beta^{2}}\right) M_{k n}(\alpha, \beta) .
\end{aligned}
$$

The invariants are

$$
\begin{aligned}
& \frac{4 \alpha^{2}+\beta^{4}+1}{2 \beta^{2}}=\frac{4 \alpha_{0}^{2}+\beta_{0}^{4}+1}{2 \beta_{0}^{2}}, \quad \kappa-\frac{\delta \varepsilon}{2 \beta}=\kappa_{0}-\frac{\delta_{0} \varepsilon_{0}}{2 \beta_{0}} \\
& \varepsilon^{2}+\frac{\delta^{2}}{\beta^{2}}=\varepsilon_{0}^{2}+\frac{\delta_{0}^{2}}{\beta_{0}^{2}}, \quad \frac{\varepsilon^{2}}{\beta^{2}}+\left(\delta-\frac{2 \alpha \varepsilon}{\beta}\right)^{2}=\frac{\varepsilon_{0}^{2}}{\beta_{0}^{2}}+\left(\delta_{0}-\frac{2 \alpha_{0} \varepsilon_{0}}{\beta_{0}}\right)^{2}
\end{aligned}
$$

by a direct calculation. Another useful identity is given by

$$
\frac{4 \alpha^{2}+\beta^{4}+1}{2 \beta^{2}} \pm 1=\frac{4 \alpha^{2}+\left(\beta^{2} \pm 1\right)^{2}}{2 \beta^{2}}=\sigma_{p}+\sigma_{x} \pm 1 .
$$

Thus all arguments of the hypergeometric functions in (6.10) are constants. Moreover, the timedependencies of the matrix elements are given by complex phase factors only:

$$
\begin{aligned}
& T_{m n}\left(\varepsilon, \frac{\delta}{\beta}, \kappa\right)=e^{2 i(m-n)\left(\gamma-\gamma_{0}\right)} T_{m n}\left(\varepsilon_{0}, \frac{\delta_{0}}{\beta_{0}}, \kappa_{0}\right), \\
& T_{m n}\left(\frac{\varepsilon}{\beta}, \delta-\frac{2 \alpha \varepsilon}{\beta}, \kappa-\frac{\alpha \varepsilon^{2}}{\beta^{2}}\right)=e^{i(n-m) t} T_{m n}\left(\frac{\varepsilon_{0}}{\beta_{0}}, \delta_{0}-\frac{2 \alpha_{0} \varepsilon_{0}}{\beta_{0}}, \kappa_{0}-\frac{\alpha_{0} \varepsilon_{0}^{2}}{\beta_{0}^{2}}\right)
\end{aligned}
$$

and

$$
M_{m n}(\alpha, \beta)=e^{-i(2 m+1)\left(\gamma-\gamma_{0}\right)} e^{-i(n+1 / 2) t} \sqrt{\frac{\beta_{0}}{\beta}} M_{m n}\left(\alpha_{0}, \beta_{0}\right)
$$


in view of the following identities

$$
\begin{aligned}
\frac{\delta}{\beta}+i \varepsilon & =\left(\frac{\delta_{0}}{\beta_{0}}+i \varepsilon_{0}\right) e^{2 i\left(\gamma-\gamma_{0}\right)} \\
\delta-\frac{2 \alpha \varepsilon}{\beta}+i \frac{\varepsilon}{\beta} & =\left(\delta_{0}-\frac{2 \alpha_{0} \varepsilon_{0}}{\beta_{0}}+i \frac{\varepsilon_{0}}{\beta_{0}}\right) e^{-i t} \\
\frac{1-\beta^{2}}{2}+i \alpha & =e^{-i t}\left(\frac{1-\beta_{0}^{2}}{2}+i \alpha_{0}\right) /\left(2 \alpha_{0} \sin t+\cos t+i \beta_{0}^{2} \sin t\right), \\
\frac{1+\beta^{2}}{2}-i \alpha & =e^{i t}\left(\frac{1+\beta_{0}^{2}}{2}-i \alpha_{0}\right) /\left(2 \alpha_{0} \sin t+\cos t+i \beta_{0}^{2} \sin t\right)
\end{aligned}
$$

and some of their complex conjugates (see also Appendix A for a complex parametrization of the Schrödinger group).

Finally, the eigenfunction expansion takes the form

$$
\psi_{n}(x, t)=\sqrt{\beta_{0}} \sum_{m=0}^{\infty} c_{m n} e^{-i(m+1 / 2) t} \Psi_{m}(x),
$$

where the time-independent coefficients are explicitly given by

$$
\begin{aligned}
c_{m n} & =\sum_{k=0}^{\infty} M_{m k}\left(\alpha_{0}, \beta_{0}\right) T_{k n}\left(\varepsilon_{0}, \frac{\delta_{0}}{\beta_{0}}, \kappa_{0}\right) \\
& =\sum_{k=0}^{\infty} T_{m k}\left(\frac{\varepsilon_{0}}{\beta_{0}}, \delta_{0}-\frac{2 \alpha_{0} \varepsilon_{0}}{\beta_{0}}, \kappa_{0}-\frac{\alpha_{0} \varepsilon_{0}^{2}}{\beta_{0}^{2}}\right) M_{k n}\left(\alpha_{0}, \beta_{0}\right)
\end{aligned}
$$

in terms of the initial data/integrals of motion (of the corresponding Ermakov-type system). Thus the total probability amplitude is connected to the product of two infinite matrices related to the Poisson and Pascal distributions.

Moreover, a combination of (4.1) and (6.21) gives the eigenfunction expansion of the TCS states. It is worth noting also that our expansion (6.21) gives an independent verification of the fact that the "missing" ${ }^{1}$ solutions (4.2) do satisfy the time-dependent Schrödinger equation (2.1). Indeed, they are written as the linear superposition (6.21)-(6.22) of standard solutions.

\section{Nonclassical Harmonic States of Motion and Photon Statistics}

A fundamental manifestation of the interaction between an atom and a field mode at resonance in an ideal cavity is the Rabi oscillations [68]. The first observation of the nonclassical radiation field of a micromaser is reported in [132] (the statistical and discrete nature of the photon field leads to collapse and revivals in the Rabi nutation [133]). Implementation of light for purposes of quantum information relies on the ability to synthesize, manipulate, and characterize various quantum states of the electromagnetic field. A review [112] covers the latest developments in quantum-state tomography of optical fields and photons (see also the references therein).

Various classes of motional states in ion traps are discussed, for example, in [101]. Our expansion formula (6.22) is consistent with statistics for the coherent, squeezed, and Fock states observed

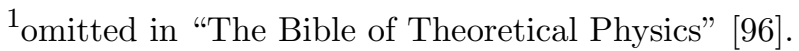


in Refs. [17] and [117] for ions and photons in a box (see also [43], [87] and [101]). A method to measure the quantum state of a harmonic oscillator through instantaneous probe-system interaction, preventing decoherence from disturbing the measurement, is proposed in [138].

7.1. Coherent States. In breakthrough experiments of the NIST group on engineering ionic states of motion, the coherent states of a single ${ }^{9} B e^{+}$ion confined in a Paul trap were produced from the ground state by a spatially uniform classical driving field and by "moving standing wave" (see [101], [117] and the references therein for details). For the data presented in [117], the authors used the first method. The Poissonian distribution with the fitted mean quantum number $\bar{n}=3.1 \pm 0.1$ was identified from Fourier analysis of Rabi oscillations. In our notation, $\alpha_{0}=0, \beta_{0}=1$, and $\bar{n}=\left(\delta_{0}^{2}+\varepsilon_{0}^{2}\right) / 2$.

Time evolution of the coherent state of cold Cs atoms was measured in [121]. For experimentally observed coherent photon states [62]; see, for example, [17] and [109].

7.2. Squeezed Vacuum and Fock States. The minimum-uncertainty squeezed state with $\gamma_{0}=$ $\delta_{0}=\varepsilon_{0}=\kappa_{0}=0$ is called the squeezed vacuum (see [43], [87], [90], and [101] when $\alpha_{0}=0$ ). Expansion (6.21) simplifies to

$$
\begin{aligned}
& \psi_{0}(x, t)=e^{i\left(\alpha(t) x^{2}+\gamma(t)\right)} \sqrt{\frac{\beta(t)}{\sqrt{\pi}}} e^{-\beta^{2}(t) x^{2} / 2} \\
& =\sqrt{\beta_{0}} \sum_{p=0}^{\infty} \frac{\sqrt{(2 p) !}}{2^{p} p !} \frac{\left(\frac{1-\beta_{0}^{2}}{2}+i \alpha_{0}\right)^{p}}{\left(\frac{1+\beta_{0}^{2}}{2}-i \alpha_{0}\right)^{p+1 / 2}} e^{-i(2 p+1 / 2) t} \Psi_{2 p}(x) .
\end{aligned}
$$

The probability distribution is restricted to the even states and given by

$$
P_{m=2 p}=\frac{(2 p) !}{\left(\sigma_{p}+\sigma_{x}+1\right)^{1 / 2} 2^{2 p-1 / 2}(p !)^{2}}\left(\frac{\sigma_{p}+\sigma_{x}-1}{\sigma_{p}+\sigma_{x}+1}\right)^{p}
$$

in terms of the variances (3.8). This is a special case of the negative binomial, or Pascal, distribution.

A vacuum squeezed state of ionic motion was created in the NIST group experiments [117] by a parametric drive at $2 \nu$ (see also [73], [101] and the references therein). The data were fitted to the vacuum state distribution (7.2) with $\sigma_{p}+\sigma_{x}=40 \pm 10$ and $\alpha_{0}=0$ (corresponding to a noise level $16 \mathrm{~dB}$ below the zero-point variance in the squeezed quadrature component; see [101] and [117] for more experimental details).

A vacuum squeezed state of motion of neutral Cs atoms was also generated in [121]. Here, the cold atom sample containes about $10^{5}$ atoms. Therefore a single image provides the full velocity distribution of the quantum state and the squeezing can be readily visualized - a set of images gives the state's time evolution (see [121] and the references therein for more details).

In a similar fashion, for the squeezed Fock state with $n=1$ and $\gamma_{0}=\delta_{0}=\varepsilon_{0}=\kappa_{0}=0$, expansion (6.21) simplifies to

$$
\psi_{1}(x, t)=\sqrt{\frac{2 \beta(t)}{\sqrt{\pi}}} e^{i\left(\alpha(t) x^{2}+3 \gamma(t)\right)} \beta(t) x e^{-\beta^{2}(t) x^{2} / 2}
$$




$$
=\frac{\beta_{0}^{3 / 2}}{\sqrt{\pi}} \sum_{p=0}^{\infty} \frac{2^{p+1} \Gamma(p+3 / 2)}{\sqrt{(2 p+1) !}} \frac{\left(\frac{1-\beta_{0}^{2}}{2}+i \alpha_{0}\right)^{p}}{\left(\frac{1+\beta_{0}^{2}}{2}-i \alpha_{0}\right)^{p+3 / 2}} e^{-i(2 p+3 / 2) t} \Psi_{2 p+1}(x) .
$$

The corresponding Pascal distribution for the odd states is given by

$$
P_{m=2 p+1}=\frac{2^{3 / 2}(3 / 2)_{p}}{\left(\sigma_{p}+\sigma_{x}+1\right)^{3 / 2} p !}\left(\frac{\sigma_{p}+\sigma_{x}-1}{\sigma_{p}+\sigma_{x}+1}\right)^{p},
$$

where $(3 / 2)_{0}=1$ and $(3 / 2)_{p}=(3 / 2)(5 / 2) \cdots(1 / 2+p)$. These squeezed Fock states were generated in [13] and their dynamics was analyzed in [121]. When $\varepsilon_{0} \neq 0$, displaced Fock states of the electromagnetic field, have been synthesized in [109] (see also the references therein).

Moreover, even/odd oscillations in the photon number distribution of the "squeezed vacuum" state, which are consequence of pair-wise generation of photon, were observed in [17], [140]. For an ideal minimum-uncertainty squeezed state zero probabilities for odd $n$ are expected, since the Hamiltonian describing the parametric process occurring inside the nonlinear crystal is quadratic in the creation and annihilation operators [43], [141]. However, the probabilities for odd photon numbers are nonzero because the squeezed state detected there is a mixed state having undergone losses inside the resonator and during the detection process which cause the distribution to smear out (see [43] and [140] for more details). The corresponding Pascal distributions (7.2) and (7.4) have different parameter values for even and odd states, which is consistent with the result of these experiments. Further details will be discussed elsewhere.

7.3. Superposition of Fock States. Generation of a coherent superposition of the ground state and the first excited Fock states of motion of cold Cs atoms in the harmonic microtraps, namely,

$$
\psi(x, t)=c_{0} e^{-i t / 2} \Psi_{0}(x)+c_{1} e^{-3 i t / 2} \Psi_{1}(x)
$$

where $c_{0}=2^{-1 / 2}$ and $c_{1}=2^{-1 / 2} e^{i \phi}$, was reported in [121] and the corresponding time evolution had been experimentally observed. This nonclassical evolution contrasts with that of a coherent state which oscillates as a classical particle without deformation (see [121] for more details).

7.4. Superfocusing of Particle Beams. An effect of proton beam focusing in a thin monocrystal film was predicted in Refs. [30], [31]. A highly collimated beam of protons $(\approx 1 \mathrm{MeV})$ entering the channel of a monocrystal film forms at a certain depth an extremely sharp $(<0.005 \mathrm{~nm})$ and relatively long (some monolayers of the crystal) focusing area where the increase of the flux density can reach up to thousand times. We shall refer to this effect as superfocusing (or Demkov's microscope). The mean effective potential of the channel can be calculated and the deflection of the fast particle within the channel can be found. In many cases the potential of the central part of the averaged channel is cylindrically symmetric and harmonic to a good approximation which can create isochronic oscillations of the ions in the plane normal to the direction of the channel. The radius of this focus can, in principle, be as small as $10^{-2} \mathrm{~nm}$.

According to Demkov's theoretical model [30], the channel average potential is independent of the channel direction $z$ and can be approximated by $\left(x^{2}+y^{2}\right) / 2$ for the transverse direction. The $z$ motion along the channel is treated classically which allows one to replace $z$ by the time $t$ setting the 
velocity equal to unity. By separation of variables, the normalized $2 D$ time-dependent Schrödinger equation,

$$
2 i \psi_{t}+\psi_{x x}+\psi_{y y}=\left(x^{2}+y^{2}\right) \psi
$$

has the following orthonormal solution:

$$
\begin{aligned}
\psi(x, y, t)= & \frac{e^{-i \arctan \left(\beta_{0}^{2} \tan t\right)}}{\sqrt{\pi}}\left(\beta_{0}^{2} \sin ^{2} t+\beta_{0}^{-2} \cos ^{2} t\right)^{-1 / 2} \\
& \times \exp \left(i \frac{\left(\beta_{0}^{2}-\beta_{0}^{-2}\right)\left(x^{2}+y^{2}\right) \sin 2 t}{4\left(\beta_{0}^{2} \sin ^{2} t+\beta_{0}^{-2} \cos ^{2} t\right)}\right) \\
& \times \exp \left(i \frac{\delta_{0}\left(2 x-\delta_{0} \sin t\right) \cos t}{2 \beta_{0}^{2}\left(\beta_{0}^{2} \sin ^{2} t+\beta_{0}^{-2} \cos ^{2} t\right)}\right) \\
& \quad \times \exp \left(-\frac{\left(x-\delta_{0} \sin t\right)^{2}+y^{2}}{2\left(\beta_{0}^{2} \sin ^{2} t+\beta_{0}^{-2} \cos ^{2} t\right)}\right)
\end{aligned}
$$

(the minimum-uncertainty squeezed state). Then

$$
\left|\psi\left(\boldsymbol{r}_{\perp}, t\right)\right|^{2}=\left(\pi\left(\beta_{0}^{2} \sin ^{2} t+\beta_{0}^{-2} \cos ^{2} t\right)\right)^{-1} \exp \left(-\frac{\left(x-\delta_{0} \sin t\right)^{2}+y^{2}}{\beta_{0}^{2} \sin ^{2} t+\beta_{0}^{-2} \cos ^{2} t}\right)
$$

with $\delta_{0}=-p_{x}$ and $\beta_{0}=R_{\min }, \beta_{0}^{-1}=R_{\mathrm{s}}$. Here, $R_{\min } R_{\mathrm{s}}=1$ in the units of original papers [30], [31].

Among other things, Demkov has predicted that the counter beams may raise an yield of nuclear reactions by orders of magnitude. He also proposed an idea of grouping of beams under action of longitudinal sawtooth fluctuations of accelerating potential. The validity of his $2 D$ harmonic channel model was confirmed by Monte Carlo computer experiments [30], [31]. (For analogous lens effects in paraxial optics; see [2], [3], [113], [164] and the references therein.)

\section{An Application to Cavity QED and Quantum Optics}

Foundations of quantum electrodynamics and quantum optics are presented in many excellent books and articles [4], [10], [11], [15], [33], [37], [45], [50], [51], [52], [53], [54], [57], [58], [62], [63], [69], [78], [82], [89], [90], [108], [119], [136], [139], [145], [148], [167], [168], [169]. Here, we suggest a modification of the radiation field operators in a perfect cavity in order to incorporate the Schrödinger symmetry group into the second quantization. Our approach gives a natural description of squeezed photons that can be created as a result of parametric amplification of quantum fluctuations in the dynamic Casimir effect [95], [172] and are registered in quantum optics [17], [112], [127].

8.1. Radiation Field Quantization in a Perfect Cavity. In the formalism of second quantization, one expands electromagnetic fields in terms of resonant modes of the particular cavity under consideration [45], [63], [82], [141], [148]. The cavity is represented by a volume $V$, bounded by a closed surface. Let $\boldsymbol{E}_{\lambda}(\boldsymbol{r}), k_{\lambda}^{2}=\omega_{\lambda}^{2} / c^{2}$ be the eigenfunctions and the eigenvalues of the corresponding boundary-value problem:

$$
\begin{aligned}
\boldsymbol{\nabla} \times \boldsymbol{\nabla} \times \boldsymbol{E}-k^{2} \boldsymbol{E} & =\mathbf{0} & & \text { in } V \\
\boldsymbol{n} \times \boldsymbol{E} & =\mathbf{0} & & \text { on } S,
\end{aligned}
$$


where $\boldsymbol{n}$ is a unit normal vector to $S$. The vector functions $\boldsymbol{H}_{\lambda}(\boldsymbol{r})$ are related to $\boldsymbol{E}_{\lambda}(\boldsymbol{r})$ by

$$
\boldsymbol{\nabla} \times \boldsymbol{E}_{\lambda}=k_{\lambda} \boldsymbol{H}_{\lambda}, \quad \boldsymbol{\nabla} \times \boldsymbol{H}_{\lambda}=k_{\lambda} \boldsymbol{E}_{\lambda} .
$$

The eigenfunctions are orthonormal in $V$ :

$$
\int_{V} \boldsymbol{E}_{\lambda} \cdot \boldsymbol{E}_{\mu} d V=\delta_{\lambda \mu}, \quad \int_{V} \boldsymbol{H}_{\lambda} \cdot \boldsymbol{H}_{\mu} d V=\delta_{\lambda \mu} .
$$

The electric and magnetic fields are expanded in the following forms

$$
\begin{aligned}
& \boldsymbol{E}(\boldsymbol{r}, t)=-\sqrt{4 \pi} \sum_{\lambda} p_{\lambda}(t) \boldsymbol{E}_{\lambda}(\boldsymbol{r}), \\
& \boldsymbol{H}(\boldsymbol{r}, t)=\sqrt{4 \pi} \sum_{\lambda} \omega_{\lambda} q_{\lambda}(t) \boldsymbol{H}_{\lambda}(\boldsymbol{r}) .
\end{aligned}
$$

The total energy is given by

$$
\mathcal{H}=\int \frac{\boldsymbol{H}^{2}+\boldsymbol{E}^{2}}{8 \pi} d V=\frac{1}{2} \sum_{\lambda}\left(p_{\lambda}^{2}+\omega_{\lambda}^{2} q_{\lambda}^{2}\right)
$$

and the Maxwell equations,

$$
\boldsymbol{\nabla} \times \boldsymbol{E}=-\frac{1}{c} \frac{\partial \boldsymbol{H}}{\partial t}, \quad \boldsymbol{\nabla} \times \boldsymbol{H}=\frac{1}{c} \frac{\partial \boldsymbol{E}}{\partial t},
$$

are equivalent to the canonical Hamiltonian equations,

$$
\frac{d q_{\lambda}}{d t}=\frac{\partial \mathcal{H}}{\partial p_{\lambda}}=p_{\lambda}, \quad \frac{d p_{\lambda}}{d t}=-\frac{\partial \mathcal{H}}{\partial q_{\lambda}}=-\omega_{\lambda}^{2} q_{\lambda},
$$

respectively.

In the second quantization, one replaces canonically conjugate coordinates and momenta by time-dependent operators $q_{\lambda}(t)$ and $p_{\lambda}(t)$ that satisfy the commutation rules

$$
\left[q_{\lambda}(t), q_{\mu}(t)\right]=\left[p_{\lambda}(t), p_{\mu}(t)\right]=0, \quad\left[q_{\lambda}(t), p_{\mu}(t)\right]=i \hbar \delta_{\lambda \mu} .
$$

The time-evolution is determined by the Heisenberg equations of motion [69]:

$$
\frac{d}{d t} p_{\lambda}(t)=\frac{i}{\hbar}\left[p_{\lambda}(t), \mathcal{H}\right], \quad \frac{d}{d t} q_{\lambda}(t)=\frac{i}{\hbar}\left[q_{\lambda}(t), \mathcal{H}\right]
$$

with appropriate initial conditions. ${ }^{2}$ (From now on, we consider a single photon cavity mode, say $v$, with frequency $\omega_{v}=1$ and use the units $c=\hbar=1$.)

8.2. Nonstandard Solutions of Heisenberg's Equations. Explicit solution of equations (8.9) for squeezed states can be found as follows

$$
p(t)=\frac{\widehat{b}(t)-\widehat{b}^{\dagger}(t)}{i \sqrt{2}}, \quad q(t)=\frac{\widehat{b}(t)+\widehat{b}^{\dagger}(t)}{\sqrt{2}} .
$$

The time-dependent annihilation $\widehat{b}(t)$ and creation $\widehat{b}^{\dagger}(t)$ operators are given by [93]

$$
\widehat{b}(t)=\frac{e^{-2 i \gamma}}{\sqrt{2}}\left(\beta x+\varepsilon+i \frac{p-2 \alpha x-\delta}{\beta}\right),
$$

\footnotetext{
${ }^{2}$ The standard form of Heisenberg's equations can be obtained by the time reversal $t \rightarrow-t$ (with $\alpha_{0} \rightarrow-\alpha_{0}$, $\gamma_{0} \rightarrow-\gamma_{0}, \delta_{0} \rightarrow-\delta_{0}$, and $\left.\kappa_{0} \rightarrow-\kappa_{0}\right)$.
} 


$$
\widehat{b}^{\dagger}(t)=\frac{e^{2 i \gamma}}{\sqrt{2}}\left(\beta x+\varepsilon-i \frac{p-2 \alpha x-\delta}{\beta}\right)
$$

in terms of the solutions (2.3)-(2.8) of Ermakov-type system. The time-independent operators $x$ and $p$ obey the canonical commutation rule $[x, p]=i$ in an abstract Hilbert space. At all times,

$$
\widehat{b}(t) \widehat{b}^{\dagger}(t)-\widehat{b}^{\dagger}(t) \widehat{b}(t)=1 \text {. }
$$

By back substitution, operators $\widehat{b}(t)$ and $\widehat{b}^{\dagger}(t)$ are solutions of the Heisenberg equation:

$$
\frac{d}{d t} \widehat{b}(t)=i[\widehat{b}(t), H], \quad \frac{d}{d t} \widehat{b}^{\dagger}(t)=i\left[\widehat{b}^{\dagger}(t), H\right],
$$

with the standard Hamiltonian

$$
H=\frac{1}{2}\left(p^{2}+x^{2}\right)
$$

subject to the following initial conditions

$$
\begin{aligned}
& \widehat{b}(0)=\frac{e^{-2 i \gamma_{0}}}{\sqrt{2}}\left(\beta_{0} x+\varepsilon_{0}+i \frac{p-2 \alpha_{0} x-\delta_{0}}{\beta_{0}}\right), \\
& \widehat{b}^{\dagger}(0)=\frac{e^{2 i \gamma_{0}}}{\sqrt{2}}\left(\beta_{0} x+\varepsilon_{0}-i \frac{p-2 \alpha_{0} x-\delta_{0}}{\beta_{0}}\right) .
\end{aligned}
$$

One may say that the transformation (8.11) allow us to incorporate the Schrödinger group of harmonic oscillator, originally found in coordinate representation [125], into a more abstract Heisenberg picture (the classical case occurs when $\beta_{0}=1$ and $\alpha_{0}=\gamma_{0}=\delta_{0}=\varepsilon_{0}=\kappa_{0}=0$ ).

8.3. Dynamic Fock Space for a Single Mode. The time-dependent quadratic invariant,

$$
\begin{aligned}
\widehat{E}(t) & =\frac{1}{2}\left[\frac{(p-2 \alpha x-\delta)^{2}}{\beta^{2}}+(\beta x+\varepsilon)^{2}\right] \\
& =\frac{1}{2}\left[\widehat{b}(t) \widehat{b}^{\dagger}(t)+\widehat{b}^{\dagger}(t) \widehat{b}(t)\right], \quad \frac{d}{d t}\langle\widehat{E}(t)\rangle=0
\end{aligned}
$$

with

$$
\frac{\partial \widehat{E}}{\partial t}+i^{-1}[\widehat{E}, H]=0, \quad H=\frac{1}{2}\left(p^{2}+x^{2}\right)
$$

extends the standard Hamiltonian/Number operator $H$ for any given real values of parameters/integrals of motion in our description of the squeezed photon state. The oscillator-type spectrum,

$$
\widehat{E}(t)\left|\psi_{n}(t)\right\rangle=\left(n+\frac{1}{2}\right)\left|\psi_{n}(t)\right\rangle
$$

can be obtained by using the modified creation and annihilation operators [4]:

$$
\begin{aligned}
& \widehat{b}(t)\left|\psi_{n}(t)\right\rangle=\sqrt{n}\left|\psi_{n-1}(t)\right\rangle, \\
& \widehat{b}^{\dagger}(t)\left|\psi_{n}(t)\right\rangle=\sqrt{n+1}\left|\psi_{n+1}(t)\right\rangle .
\end{aligned}
$$

With a proper choice of the global phase, the latter eigenstates of dynamical invariant satisfy the time-dependent Schrödinger equation in an abstract Hilbert space [56], [93].

For the "minimum-uncertainty squeezed states", one gets

$$
\widehat{b}(t)\left|\psi_{0}(t)\right\rangle=0
$$


with

$$
\left\langle\psi_{0}(t)|H| \psi_{0}(t)\right\rangle=\frac{1+4 \alpha_{0}^{2}+\beta_{0}^{4}}{4 \beta_{0}^{2}}+\frac{\left(2 \alpha_{0} \varepsilon_{0}-\beta_{0} \delta_{0}\right)^{2}+\varepsilon_{0}^{2}}{2 \beta_{0}^{2}} \geq \frac{1}{2}
$$

in the Schrödinger picture. The generalized coherent (or TCS's) states are given by

$$
\widehat{b}(t)|\psi(t)\rangle=\zeta|\psi(t)\rangle
$$

for an arbitrary complex $\zeta \neq 0$.

8.4. Expectation Values and Variances for Field Oscillators. The noncommuting electric $\boldsymbol{E}(\boldsymbol{r}, t)$ and magnetic $\boldsymbol{H}(\boldsymbol{r}, t)$ field operators are given by equations (8.4) and (8.10)-(8.11) for a squeezed photon in the Heisenberg picture, which provides a more direct analogy between quantum and classical physics [68]. The electromagnetic radiation mode in a cavity resonator is analogous to a harmonic oscillator [70]. In the Schrödinger picture, all previous results on the minimumuncertainty squeezed states can be reproduced for the field oscillators in an operator QED-style. For a single mode with $\omega_{v}=1$,

$$
\begin{aligned}
& \langle\boldsymbol{E}(\boldsymbol{r}, t)\rangle=-\sqrt{4 \pi} \boldsymbol{E}_{v}(\boldsymbol{r})\left\langle\psi_{n}(t)|p| \psi_{n}(t)\right\rangle, \\
& \langle\boldsymbol{H}(\boldsymbol{r}, t)\rangle=\sqrt{4 \pi} \boldsymbol{H}_{v}(\boldsymbol{r})\left\langle\psi_{n}(t)|x| \psi_{n}(t)\right\rangle,
\end{aligned}
$$

where equations (3.1)-(3.2) hold. The corresponding variances are given (up to a normalization) by equations (A.4)-(A.5) of Ref. [105].

The minimum-uncertainty squeezed states are identified in quantum optics [34], [70], [65], [81], [87], [101], [146], [149], [131], [136], [174] and in state tomography [18], [48], [102], [112]. They are also important in the dynamical Casimir effect [35], [36], [37], [45], [59], [93], [95], [115], [172], and [173], where the photon squeezing occurs as a result of a "parametric excitation" of vacuum oscillations.

\section{An Important Variance}

The Hamiltonian $H=\left(p^{2}+x^{2}\right) / 2$ can be rewritten in terms of the creation and annihilation operators (2.12) as follows:

$$
\begin{aligned}
H= & \left(\frac{4 \alpha^{2}-\beta^{4}+1}{4 \beta^{2}}-i \alpha\right) \widehat{a}^{2}(t)+\left(\frac{4 \alpha^{2}-\beta^{4}+1}{4 \beta^{2}}+i \alpha\right) \widehat{a}^{\dagger}(t)^{2} \\
& +\frac{4 \alpha^{2}+\beta^{4}+1}{4 \beta^{2}}\left[\widehat{a}(t) \widehat{a}^{\dagger}(t)+\widehat{a}^{\dagger}(t) \widehat{a}(t)\right] \\
& +\sqrt{2}\left[\frac{\alpha}{\beta}\left(\delta-\frac{2 \alpha \varepsilon}{\beta}\right)-\frac{\varepsilon}{2 \beta^{2}}-\frac{i \beta}{2}\left(\delta-\frac{2 \alpha \varepsilon}{\beta}\right)\right] \widehat{a}(t) \\
& +\sqrt{2}\left[\frac{\alpha}{\beta}\left(\delta-\frac{2 \alpha \varepsilon}{\beta}\right)-\frac{\varepsilon}{2 \beta^{2}}+\frac{i \beta}{2}\left(\delta-\frac{2 \alpha \varepsilon}{\beta}\right)\right] \widehat{a}^{\dagger}(t) \\
& +\frac{1}{2}\left(\delta-\frac{2 \alpha \varepsilon}{\beta}\right)^{2}+\frac{\varepsilon^{2}}{2 \beta^{2}}
\end{aligned}
$$

and by definition:

$$
\text { Var } H=\left\langle(H-\langle H\rangle)^{2}\right\rangle=\left\langle H^{2}\right\rangle-\langle H\rangle^{2}
$$


Then a direct Mathematica calculation results in

$$
\begin{aligned}
& \operatorname{Var} H=\frac{\left(4 \alpha_{0}^{2}+\left(\beta_{0}^{2}+1\right)^{2}\right)\left(4 \alpha_{0}^{2}+\left(\beta_{0}^{2}-1\right)^{2}\right)}{8 \beta_{0}^{4}}\left[\left(n+\frac{1}{2}\right)^{2}+\frac{3}{4}\right] \\
& +\left[\frac{\left(4 \alpha_{0}^{2}+\beta_{0}^{4}+1\right)\left(\left(2 \alpha_{0} \varepsilon_{0}-\beta_{0} \delta_{0}\right)^{2}+\varepsilon_{0}^{2}\right)}{\beta_{0}^{4}}-\left(\varepsilon_{0}^{2}+\frac{\delta_{0}^{2}}{\beta_{0}^{2}}\right)\right]\left(n+\frac{1}{2}\right)
\end{aligned}
$$

for the wavefunctions (4.2) in terms of the invariants (6.11)-(6.12). (These calculations can be performed in pure operator form with the help of standard relations (1.15) of Ref. [105]; see also (8.19).) In terms of the variances,

$$
\begin{aligned}
\operatorname{Var} H= & \frac{1}{2}\left[\left(\sigma_{p}+\sigma_{x}\right)^{2}-1\right]\left[\left(n+\frac{1}{2}\right)^{2}+\frac{3}{4}\right] \\
& +2\left[\sigma_{p}\langle p\rangle^{2}+2 \sigma_{p x}\langle p\rangle\langle x\rangle+\sigma_{x}\langle x\rangle^{2}\right]\left(n+\frac{1}{2}\right),
\end{aligned}
$$

where $\sigma_{p}, \sigma_{x}$, and $\sigma_{p x}$ are given by (3.7). When $n=0$, this formula is consistent with the variance of the number operator derived for a generic Gaussian Wigner function in Ref. [43].

A similar expression holds for the TCS states. Computational details are left to the reader.

\section{A Conclusion}

In this paper, we review some properties of the nonclassical states of harmonic motion which were originally found in [116] (in coordinate representation) and have been rediscovered recently in [105]. They are useful in applications to cavity QED, quantum optics, and in channeling scattering [30]. In particular, the minimum-uncertainty squeezed states are studied in detail. Expansions in the Fock states are established and their relations with experimentally observed photon statistics are briefly discussed. In the method of second quantization, a modification of the radiation field operators for squeezed photons in a perfect cavity is suggested with the help of a nonstandard solution of Heisenberg's equation of motion. These results may be of interest to everyone who studies introductory quantum mechanics and quantum optics.

Acknowledgments. We would like to thank Viktor Dodonov, Mark Faifman, Geza Giedke, John R. Klauder, Alex Mahalov, Vladimir I. Man'ko, and Andreas Ruffing for valuable discussions. The authors are grateful to the referees for valuable suggestions. This research is supported in part by the National Science Foundation-Enhancing the Mathematical Sciences Workforce in the 21st Century (EMSW21), award \# 0838705; the Alfred P. Sloan Foundation-Sloan National Pipeline Program in the Mathematical and Statistical Sciences, award \# LTR 05/19/09.

\section{Appendix A. A Complex Parametrization of the Schrödinger Group}

The Ansatz

$$
\psi(x, t)=\sqrt{\beta(t)} e^{i S(x, t)} \chi(\xi, \tau), \quad S=\alpha(t) x^{2}+\delta(t) x+\kappa(t),
$$


where relations (2.3)-(2.8) hold, transforms the time-dependent Schrödinger equation (2.1) into itself:

$$
2 i \psi_{t}+\psi_{x x}-x^{2} \psi=e^{i S} \beta^{5 / 2}\left(2 i \chi_{\tau}+\chi_{\xi \xi}-\xi^{2} \chi\right)=0
$$

with respect to the new variables $\xi=\beta(t) x+\varepsilon(t)$ and $\tau=-\gamma(t)$. This transformation is known as the Schrödinger group for linear harmonic oscillator [125].

Let us introduce the following complex-valued function:

$$
z=c_{1} e^{i t}+c_{2} e^{-i t}, \quad z^{\prime \prime}+z=0
$$

where by definition

$$
\begin{gathered}
c_{1}=\left(1+\beta_{0}^{2}\right) / 2-i \alpha_{0}, \quad c_{2}=\left(1-\beta_{0}^{2}\right) / 2+i \alpha_{0} \\
\left(c_{1}+c_{2}=1, \quad\left|c_{1}\right|^{2}-\left|c_{2}\right|^{2}=\beta_{0}^{2}\right),
\end{gathered}
$$

and

$$
c_{3}=\frac{\delta_{0}}{\beta_{0}}-i \varepsilon_{0} .
$$

Then equations (2.3)-(2.8) can be rewritten in a compact form in terms of our complex parameters $c_{1}, c_{2}$, and $c_{3}$. Indeed, with the help of identities (6.17)-(6.20), one gets

$$
|z|=\left(\left|c_{1}\right|^{2}+c_{1} c_{2}^{*} e^{2 i t}+c_{1}^{*} c_{2} e^{-2 i t}+\left|c_{2}\right|^{2}\right)^{1 / 2}
$$

and

$$
\begin{aligned}
\alpha & =i \frac{c_{1} c_{2}^{*} e^{2 i t}-c_{1}^{*} c_{2} e^{-2 i t}}{2|z|^{2}}, \\
\beta & =\frac{\beta_{0}}{|z|}= \pm \frac{\sqrt{\left|c_{1}\right|^{2}-\left|c_{2}\right|^{2}}}{|z|} \\
\gamma & =\gamma_{0}-\frac{1}{2} \arg z \\
\delta & =\frac{\beta_{0}}{2|z|}\left(c_{3} e^{i \arg z}+c_{3}^{*} e^{-i \arg z}\right), \\
\varepsilon & =\frac{i}{2}\left(c_{3} e^{i \arg z}-c_{3}^{*} e^{-i \arg z}\right), \\
\kappa & =\kappa_{0}-\frac{i}{8}\left[c_{3}^{2}\left(1-e^{2 i \arg z}\right)-c_{3}^{* 2}\left(1-e^{-2 i \arg z}\right)\right] .
\end{aligned}
$$

The inverse relations between the essential, real and complex, parameters are given by

$$
\begin{gathered}
\alpha_{0}=\frac{i}{2}\left(c_{1} c_{2}^{*}-c_{1}^{*} c_{2}\right), \quad \beta_{0}= \pm \sqrt{\left|c_{1}\right|^{2}-\left|c_{2}\right|^{2}}, \\
\delta_{0}= \pm \frac{1}{2} \sqrt{\left|c_{1}\right|^{2}-\left|c_{2}\right|^{2}}\left(c_{3}+c_{3}^{*}\right), \quad \varepsilon_{0}=\frac{i}{2}\left(c_{3}-c_{3}^{*}\right) .
\end{gathered}
$$

These formulas (A.7)-(A.12) provide a complex parametrization of the Schrödinger group for the simple harmonic oscillator originally found in Ref. [125] (see also [104], [105] and the references therein). A similar parametrization for the wavefunctions (4.2) was used in Ref. [40] (see [67] and [93] for an extension to generalized harmonic oscillators). 


\section{REFERENCES}

[1] J. Abadie et al., A gravitational wave observatory operating beyond the quantum shot-noise limit, Nature Physics 7 (2011), 962-965.

[2] E. G. Abramochkin and V. G. Volostnikov, Spiral light beams, Physics-Uspekhi 47 (2004) \# 12, 1177-1203.

[3] G. P. Agrawal, A. K. Ghatak, and C. L. Mehtav, Propagation of a partially coherent beam through selfoc fibers, Opt. Comm. 12 (1974) \# 3, 333-337.

[4] A. Akhiezer and V. B. Berestetskii, Quantum Electrodynamics, Interscience Publishers, New York, 1965.

[5] R. L. Anderson, S. Kumei, and C. E. Wulfman, Invariants of the equations of wave mechanics. I, Rev. Mex. Fís. 21 (1972), 1-33.

[6] R. L. Anderson, S. Kumei, and C. E. Wulfman, Invariants of the equations of wave mechanics. II One-particle Schrödinger equations, Rev. Mex. Fís. 21 (1972), 35-57.

[7] V. G. Bagrov, V. V. Belov and I. M. Ternov, Quasiclassical trajectory-coherent states of a particle in an arbitrary electromagnetic field, J. Math. Phys. 24 (1983) \# 12, 2855-2859.

[8] W. N. Bailey, Some integrals involving Hermite polynomials, J. London Math. Soc. 23 (1948) \# 4, $291-297$.

[9] V. V. Belov and A. G. Karavaev, Higher approximations for quasiclassical trajectory-coherent states, Izvestiya Vysshikh Uchebynkh Zavedenij Fizika, 31 (1987) \# 10, 14-18 [in Russian]; see also English transl.: Sov. Phys. Journal 1989, 30 \#10, 819-822.

[10] V. B. Berestetskii, E. M. Lifshitz, and L. P. Pitaevskii, Relativistic Quantum Theory, Pergamon Press, Oxford, 1971.

[11] I. Białynicki-Birula and Z. Białynicki-Birula, Quantum Electrodynamics, Pergamon Press Ltd. and PWNPolish Scientific Publishers, Oxford, New York, Toronto, Sydney, Warszawa, 1975.

[12] N. D. Birrell and P. C. W. Davies, Quantum Fields in Curved Space, Cambridge University Press, Cambridge, 1982 .

[13] I. Bouchoule, H. Perrin, A. Kuhn, M. Morinaga, and C. Salomon, Neutral atoms prepared in Fock states of a one-dimensional harmonic potential, Phys. Rev. A 59 (1999) \# 1, R8-R11.

[14] A. Buchleitner, D. Delandea, and J. Zakrzewski, Non-dispersive wave packets in periodically driven quantum systems, Phys. Rep. 368 (2002), 409-547.

[15] N. N. Bogoliubov and D. V. Shirkov, Introduction to the Theory of Quantized Fields, third edition, John Wiley \& Sons, New York, Chichester, Brisbane, Toronto, 1980.

[16] C. P. Boyer, R. T. Sharp, and P. Winternitz, Symmetry breaking interactions for the time dependent Schrödinger equation, J. Math. Phys. 17 (1976) \# 8, 1439-1451.

[17] G. Breitenbach, S. Schiller, and J. Mlynek, Measurement of the quantum states of squeezed light, Nature $\mathbf{3 8 7}$ (1997) May 29, 471-475.

[18] V. N. Chernega and V. I. Man'ko, Probability representation and state-extended uncertainty relations, Journal of Russian Laser Research 32 (2011) \# 2, 125-129.

[19] J. I. Cirac, Entanglement in many-body quantum systems, arXiv:1205.3742v1 [quant-ph] 16 May 2012.

[20] J. I. Cirac, R. Blatt, A. S. Parkins, and P. Zoller, Preparation of Fock states by observing of quantum jumps in an ion trap, Phys. Rev. Lett. 70 (1993) \# 6, 762-765.

[21] J. I. Cirac, R. Blatt, A. S. Parkins, and P. Zoller, Quantum collapse and revival in the motion of a single trapped ion, Phys. Rev. A. 49 (1994) \# 2, 1202-1207.

[22] J. I. Cirac, R. Blatt, and P. Zoller, Nonclassical states of motion in a three-dimensional ion trap by adiabatic passage, Phys. Rev. A. 49 (1994) \# 5, R3174-R3177.

[23] J. I. Cirac, A. S. Parkins, R. Blatt, and P. Zoller, "Dark" states of the motion of a trapped ion, Phys. Rev. Lett. Lett. 70 (1993) \# 5, 556-559.

[24] J. I. Cirac and P. Zoller, Quantum computations with cold trapped ions, Phys. Rev. Lett. 74 (1995) \# 20, 4091-4094.

[25] S. M. Chumakov, A. B. Klimov, and M. Kozierowski, From the Jaynes-Cummings model to collective interactions, in: Theory of Nonclassical States of Light (V. V. Dodonov and V. I. Man'ko, Editors), Taylor \& Francis, London and New York, 2003.

[26] R. J. Cook, D. G. Shankland, and A. L. Wells, Quantum theory of particle motion in a rapidly oscillating field, Phys. Rev. A 31 (1985) \# 2, 564-567.

[27] R. Cordero-Soto, R. M. López, E. Suazo, and S. K. Suslov, Propagator of a charged particle with a spin in uniform magnetic and perpendicular electric fields, Lett. Math. Phys. 84 (2008) \# 2-3, 159-178. 
[28] R. Cordero-Soto, E. Suazo, and S. K. Suslov, Models of damped oscillators in quantum mechanics, J. Phys. Math. 1 (2009), S090603 (16 pages).

[29] R. Cordero-Soto, E. Suazo, and S. K. Suslov, Quantum integrals of motion for variable quadratic Hamiltonians, Ann. Phys. 325 (2010) \# 9, 1884-1912.

[30] Yu. N. Demkov, Channeling, superfocusing, and nuclear reactions, Physics of Atomic Nuclei, 72 (2009) \# 5, 779-785.

[31] Yu. N. Demkov and J. D. Meyer, A sub-atomic microscope, superfocusing in channeling and close encounter atomic and nuclear reactions, Eur. Phys. J. B 42 (2004), 361-365.

[32] F. Diedrich, J. C. Bergquist, W. M. Itano, and D. J. Wineland, Laser cooling to the zero-point energy of motion, Phys. Rev. Lett. 62 (1989) \# 4, 403-406.

[33] P. A. M. Dirac, The Principles of Quantum Mechanics, third edition, Clarendon Press, Oxford, 1947.

[34] V. V. Dodonov, 'Nonclassical' states in quantum optics: a 'squeezed' review of the first 75 years, J. Opt. B: Quantum Semiclass. Opt. 4 (2002), R1-R33.

[35] V. V. Dodonov, Current status of dynamical Casimir effect, Physica Scripta 82 (2010) \# 3, 038105 (10 pp).

[36] V. V. Dodonov, A. B. Klimov, and V. I. Man'ko, Generation of squeezed states in a resonator with a moving wall, Phys. Lett. A. 149 (1990) \# 4, 225-228.

[37] V. V. Dodonov, A. B. Klimov, and D. E. Nikonov, Quantum phenomena in nonstationary media, Phys. Rev. A. 47 (1993) \# 5, 4422-4429.

[38] V. V. Dodonov, A. B. Kurmyshev, and V. I. Man'ko, Generalized uncertainty relation and correlated coherent states, Phys. Lett. A. 79 (1980) \# 2,3, 150-152.

[39] V. V. Dodonov, I. A. Malkin, and V. I. Man'ko, Integrals of motion, Green functions, and coherent states of dynamical systems, Int. J. Theor. Phys. 14 (1975) \# 1, 37-54.

[40] V. V. Dodonov and V. I. Man'ko, Coherent states and the resonance of a quantum damped oscillator, Phys. Rev. A 20 (1979) \# 2, 550-560.

[41] V. V. Dodonov and V. I. Man'ko, Invariants and correlated states of nonstationary quantum systems, in: Invariants and the Evolution of Nonstationary Quantum Systems, Proceedings of Lebedev Physics Institute, vol. 183, pp. 71-181, Nauka, Moscow, 1987 [in Russian]; English translation published by Nova Science, Commack, New York, 1989, pp. 103-261.

$[42]$ V. V. Dodonov and V. I. Man'ko, "Nonclassical" states in quantum optics: brief historical review, in: Theory of Nonclassical States of Light (V. V. Dodonov and V. I. Man'ko, Editors), Taylor \& Francis, London and New York, 2003.

[43] V. V. Dodonov, O. V. Man'ko, and V. I. Man'ko, Photon distribution for one-mode mixed light with a generic gaussian Wigner function, Phys. Rev. A 49 (1994), 2993-3001.

[44] T. J. Dunn, I. A. Walmsley, and S. Mukamel, Experimental determination of the quantum-mechanical state of a molecular vibrational mode using fluorescence tomography, Phys. Rev. Lett. 74 (1995), 884-887.

[45] S. M. Dutra, Cavity Quantum Electrodynamics : The Strange Theory of Light in a Box, Hoboken, NJ, USA: Wiley, 2005.

[46] T. Eberle et al., Quantum enhancement of the zero-area Sagnac interferometer topology for gravitational wave detection, Phys. Rev. Lett. 104 (2010), 251102 (4 pages).

[47] P. Ehrenfest, Bemerkung über die angenäherte Gültigkeit der klassischen Mechanik innerhalb der Quantenmechanik, Zeitschrift für Physik A 45 (1927), 455-457.

[48] C. Eichler, D. Bozyigit, C. Lang, M. Baur, L. Steffen, J. M. Fink, S. Filipp, and A. Wallraff, Observation of two-mode squeezing in the microwave frequency domain, Phys. Rev. Lett. 107 (2011) , 113601 (5 pages).

[49] V. P. Ermakov, Second-order differential equations. Conditions of complete integrability, Universita Izvestia Kiev, Series III 9 (1880), 1-25; see also Appl. Anal. Discrete Math. 2 (2008) \# 2, 123-145 for English translation of Ermakov's original paper.

[50] R. P. Feynman, The Theory of Fundamental Processes, Perseus Books Publishing, Cambridge, Massachusetts, 1998.

[51] R. P. Feynman, QED: The Strange Theory of Light and Matter, Princeton University Press, Princeton and Oxford, 2006.

[52] R. P. Feynman and A. R. Hibbs, Quantum Mechanics and Path Integrals, McGraw-Hill, New York, 1965.

[53] E. Fermi, Quantum theory of radiation, Rev. Mod. Phys. 4 (1932), 87-132. 
[54] E. Fermi, Notes on Quantum Mechanics, Phoenix Science Series, The University of Chicago Press, Chicago and London, 1961.

[55] S. Flügge, Practical Quantum Mechanics, Springer-Verlag, Berlin, 1999.

[56] V. Fock, On the relation between the integrals of the quantum mechanical equations of motion and the Schrödinger wave equation, Zs. Phys. 49 (1928) \# 5-6, 323-338; reprinted in: V. A. Fock, Selected Works: Quantum Mechanics and Quantum Field Theory, (L. D. Faddeev, L. A. Khalfin, and I. V. Komarov, Eds.), Chapman \& Hall/CRC, Boca Raton, London, New York, Washington, D. C., 2004, pp. 33-49.

[57] V. Fock, Configuration space and second quantization, Zs. Phys. 75 (1932) \# 9-10, 622-647; reprinted in: V. A. Fock, Selected Works: Quantum Mechanics and Quantum Field Theory, (L. D. Faddeev, L. A. Khalfin, and I. V. Komarov, Eds.), Chapman \& Hall/CRC, Boca Raton, London, New York, Washington, D. C., 2004, pp. 191-220.

[58] V. Fock, On quantum electrodynamics, Phys. Zs. Sowjetunion 6 (1934), 425-469; reprinted in: V. A. Fock, Selected Works: Quantum Mechanics and Quantum Field Theory, (L. D. Faddeev, L. A. Khalfin, and I. V. Komarov, Eds.), Chapman \& Hall/CRC, Boca Raton, London, New York, Washington, D. C., 2004, pp. 331-368.

[59] T. Fujii, Sh. Matsuo, N. Hatakenaka, S. Kurihara, and A. Zeilinger, Quantum circuit analog of the dynamical Casimir effect, Phys. Rev. B. 84 (2011) \# 17, 174521 (9 pages).

[60] B. M. Garraway, Extended Gaussian wavepacket dynamics, J. Phys. B: At. Mol. Opt. Phys. 33 (2000), 44474467.

[61] I. Gerhardt, G. Wrigge, G. Zumofen, J. Hwang, A. Renn, and V. Sandoghdar, Coherent state preparation and observation of Rabi oscillations in a single molecule, Phys. Rev. A. 79 (2009) \# 1, 011402(R) (4 pages).

[62] R. J. Glauber, Quantum Theory of Optical Coherence: Selected Papers and Lectures, WILEY-VCH Verlag GmbH \& Co. KGaA, Weinheim, 2007.

[63] R. J. Glauber and M. Lewenstein, Quantum optics of dielectric media, Phys. Rev. A 43 (1991) \# 1, $467-491$.

[64] I. I. Gol'dman and V. D. Krivchenkov, Problems in Quantum Mechanics, Dover, New York, 1993.

[65] J. Guerrero, and F. F. López-Ruiz, V. Aldaya, and F. Cossio, Harmonic states for the free particle, J. Phys. A: Math. Theor. 44 (2011), 445307 (16pp); see also arXiv:1010.5525v3 [quant-ph] 1 Jul 2011.

[66] C. H. Hagen, Scale and conformal transformations in Galilean-covariant field theory, Phys. Rev. D 5 (1972) \# 2, $377-388$.

[67] G. Harari, Ya. Ben-Aryeh, and Ady Mann, Propagator for the general time-dependent harmonic oscillator with application to an ion trap, Phys. Rev. A 84 (2011) \# 6, 062104 (4 pages).

[68] S. Haroche J.-M. Raimond, Exploring the Quantum: Atoms, Cavities, and Photons, Oxford University Press, Oxford, 2006.

[69] W. Heisenberg, The Physical Principles of the Quantum Theory, University of Chicago Press, Chicago, 1930; Dover, New York, 1949.

[70] R. W. Henry and S. C. Glotzer, A squeezed-state primer, Am. J. Phys. 56 (1988) \# 4, 318-328.

[71] S. W. Hawking, Black hole explosions?, Nature, London 248 (1974), 30-31.

[72] S. W. Hawking, Particle creation by black holes, Commun. Math. Phys. 43 (1975) \# 3, $199-220$.

[73] D. J. Heinzen and D. J. Wineland, Quantum-limited cooling and detection of radio-frequency oscillations by laser-cooled ions, Phys. Rev. A (1990) \# 5, 2977-2994.

[74] M. Hillery, R. F. O'Connel, M. O. Scully, and E. P. Wigner, Distribution functions in physics: fundamentals, Phys. Rep. 106 (1953) \# 3, 121-167.

[75] J. N. Hollenhorst, Quantum limits on resonant-mass gravitational-radiation detectors, Phys. Rev. D (1979) \# 6 , 1669-1679.

[76] K. Husimi, Miscellanea in elementary quantum mechanics: I-II, Prog. Theor. Phys. 9 (1953) \# 3, 238-244; Prog. Theor. Phys. 9 (1953) \# 4, 381-402.

[77] K. Husimi and M. Ôtuka, Miscellanea in elementary quantum mechanics: III, Prog. Theor. Phys. 10 (1953) \# 2, 173-190.

[78] C. Itzykson and J-B. Zuber, Quantum Field Theory, Dover Publications, New York, 2005.

[79] R. Jackiw, Dynamical symmetry of the magnetic monopole, Ann. Phys. 129 (1980), 183-200.

[80] T. A. Jacobson, Introduction to quantum fields in curved spacetime and the Hawking effect, arXiv:0308048v3 [gr-qc] 9 April 2004.

[81] N. Jain, S. R. Huisman, E. Bimbard, and A. I. Lvovsky, A bridge between the single-photon and squeezed-vacuum states, Optics Express 18 (2010) \# 17, 18254 (6 pages). 
[82] E. T. Jaynes and F. W. Cummings, Comparison of quantum and semiclassical radiation theories with application to the beam maser, Proc. IEEE. 51 (1963) \# 1, 89-109.

[83] P. S. Jessen, C. Gerz, P. D. Lett, W. D. Phillips, S. L. Rolston, R. J. C. Spreeuw, and C. I. Westbrook, Observation of quantized motion of Rb atoms in an optical field, Phys. Rev. Lett. 69 (1992) \# 1, 49-52.

[84] M. Johanning, A. F. Varón, and Ch. Wunderlich, Quantum simulations with cold trapped ions, J. Phys. B: At. Mol. Opt. Phys. 42 (2009), 154009 (27pp).

[85] E. G. Kalnins and W. Miller, Lie theory and separation of variables. 5. The equations $i U_{t}+U_{x x}=0$ and $i U_{t}+U_{x x}-c / x^{2} U=0$, J. Math. Phys. 15 (1974) \# 10, 1728-1737.

[86] E. H. Kennard, Zur Quantenmechanik einfacher Bewegungstypen, Zeitschrift für Physik 44 (1927) \# 4-5, $326-352$.

[87] M. S. Kim, F. A. M. de Oliveira, and P. L. Knight, Properties of squeezed number states and squeezed thermal states, Phys. Rev. A 40 (1989) \# 5, 2494-2503.

[88] J. R. Klauder, The design of radar signals having both high range resolution and high velocity resolution, The Bell system technical journal 39 (1960) \# 4, 809-820.

[89] J. R. Klauder, Enhanced quantization: a primer, J. Phys. A: Math. Theor. 45 (2012), 285304 (8 pages).

[90] J. R. Klauder and E. C. G. Sudarshan, Fundamentals of Quantum Optics, W. A. Benjamin, Inc., New York, Amsterdam, 1968.

[91] C. Koutschan, http://hahn.la.asu.edu/ suslov/curres/index.htm; see Mathematica notebook: Koutschan.nb; see also http://www.risc.jku.at/people/ckoutsch/pekeris/

[92] C. Koutschan and D. Zeilberger. The 1958 Pekeris-Accad-WEIZAC Ground-Breaking Collaboration that computed Ground States of Two-Electron Atoms (and its 2010 Redux), Math. Intelligencer 33 (2011) \# 2, $52-57$.

[93] C. Krattenthaler, S. I. Kryuchkov, A. Mahalov, and S. K. Suslov, On the problem of electromagnetic-field quantization, arXiv:1301.7328v2 [math-ph] 9 Apr 2013.

[94] S. I. Kryuchkov, S. K. Suslov and J. M. Vega-Guzmán, http://hahn.la.asu.edu/〜 suslov/curres/index.htm; see Mathematica notebook: WignerSummary.nb.

[95] P. Lähteenmäki, G. S. Paraoanu, J. Hassel, and P. J. Hakonen, Dynamical Casimir effect in a Josephson metamaterial, arXiv:1111.5608v2 [cond-mat.mes-hall] 1 Dec 2011.

[96] L. D. Landau and E. M. Lifshitz, Quantum Mechanics: Nonrelativistic Theory, Pergamon Press, Oxford, 1977.

[97] N. Lanfear and S. K. Suslov, The time-dependent Schrödinger equation, Riccati equation and Airy functions, arXiv:0903.3608v5 [math-ph] 22 Apr 2009.

[98] N. Lanfear, R. M. López, and S. K. Suslov, Exact wave functions for generalized harmonic oscillators, Journal of Russian Laser Research 32 (2011) \# 4, 352-361; see also arXiv:11002.5119v2 [math-ph] 20 Jul 2011.

[99] P. G. L. Leach and K. Andriopoulos, The Ermakov equation: a commentary, Appl. Anal. Discrete Math. 2 (2008) \# 2, 146-157.

[100] C. R. Leavens, R. Sala Mayato, On constructing the wave function of a quantum particle from its Wigner phase-space distribution function, Phys. Lett. A 280 (2001), 163-172.

[101] D. Leibfried, R. Blatt, C. Monroe, and D. Wineland, Quantum dynamics of single trapped ions, Rev. Mod. Phys. 75 (2003) \# 1, 281-324.

[102] U. Leonhard and H. Paul, Measuring the quantum state of light, Prog. Quant. Electr. 19 (1995), 89-130.

[103] R. M. López and S. K. Suslov, The Cauchy problem for a forced harmonic oscillator, Revista Mexicana de Física, 55 (2009) \# 2, 195-215; see also arXiv:0707.1902v8 [math-ph] 27 Dec 2007.

[104] R. M. López, S. K. Suslov, and J. M. Vega-Guzmán, Reconstracting the Schrödinger groups, Physica Scripta 87 (2013) \# 3, 038112 (6 pages); see also On the harmonic oscillator group, arXiv:1111.5569v2 [math-ph] 4 Dec 2011.

[105] R. M. López, S. K. Suslov, and J. M. Vega-Guzmán, On a hidden symmetry of quantum harmonic oscillators, Journal of Difference Equations and Applications, 19 (2013) \# 4, 543-554: http://www.tandfonline.com/doi/abs/10.1080/10236198.2012.658384\#preview; see also arXiv:1112.2586v2 [quant-ph] 2 Jan 2012.

[106] R. M. López, S. K. Suslov, and J. M. Vega-Guzmán, http://hahn.la.asu.edu/〜suslov/curres/index.htm; see Mathematica notebook: HarmonicOscillatorGroup.nb.

[107] R. D. Lord, Some integrals involving Hermite polynomials, J. London Math. Soc. 24 (1948) \# 2, 101-112.

[108] W. H. Louisell, Quantum Statistical Properties of Radiation, Wiley, New York, 1973. 
[109] A. I. Lvovsky and S. A. Babichev, Synthesis and tomographic characterization of the displaced Fock state of light, Phys. Rev. 66 (2002), 011801(R) (4 pages).

[110] A. I. Lvovsky, H. Hansen, T. Aichele, O. Benson, J. Mlynek, and S. Schiller, Quantum state reconstruction of the single-photon Fock state, Phys. Rev. Lett. 87 (2001) \# 5, 050402 (4 pages).

[111] A. I. Lvovsky and J. Mlynek, Quantum-optical catalysis: generating nonclassical states of light by means of linear optics, Phys. Rev. Lett. 88 (2002) \# 25, 250401 (4 pages).

[112] A. I. Lvovsky and M. G. Raymer, Continuous-variable optical quantum-state tomography, Rev. Mod. Phys. 81 (2009), January-March, 299-332.

[113] A. Mahalov and S. K. Suslov, Solution of paraxial wave equation for inhomogeneous media in linear and quadratic approximation, submitted.

[114] I. A. Malkin and V. I. Man'ko, Dynamical Symmetries and Coherent States of Quantum System, Nauka, Moscow, 1979 [in Russian].

[115] V. I. Man'ko, The Casimir effect and quantum vacuum generator, Journal of Soviet Laser Research 12 (1991), 383-385.

[116] M. E. Marhic, Oscillating Hermite-Gaussian wave functions of the harmonic oscillator, Lett. Nuovo Cim. 22 (1978) \# 8, 376-378.

[117] D. M. Meekhof, C. Monroe, B. E. King, W. M. Itano, and D. J. Wineland, Generation of nonclassical motional states of a trapped atom, Phys. Rev. Lett. 76 (1996) \# 11, 1796-1799.

[118] C. Monroe, D. M. Meekhof, B. E. King, W. M. Itano, and D. J. Wineland, Demonstration of a fundamental quantum logic gate, Phys. Rev. Lett. 75 (1995) \# 25, 4714-4717.

[119] E. Merzbacher, Quantum Mechanics, third edition, John Wiley \& Sons, New York, 1998.

[120] W. Miller, Jr., Symmetry and Separation of Variables, Encyclopedia of Mathematics and Its Applications, Vol. 4, Addison-Wesley Publishing Company, Reading etc, 1977.

[121] M. Morinaga, I. Bouchoule, J.-C. Karam, and C. Salomon, Manipulation of motional quantum states of neutral atoms, Phys. Rev. Lett. 83 (1999) \# 20, 4037-4040.

[122] J. E. Moyal, Quantum mechanics as a statistical theory, Proc. Camb. Phil. Soc. 49 (1947), 99-124.

[123] P. D. Nation, J. R. Johansson, M. P. Blencowe, and F. Nori, Stimulationg uncertainty: Amplifying the quantum vacuum with superconducting circuits, Rev. Mod. Phys. 84 (2012), January-March, 1-24.

[124] U. Niederer, The maximal kinematical invariance group of the free Schrödinger equations, Helv. Phys. Acta 45 (1972), 802-810.

[125] U. Niederer, The maximal kinematical invariance group of the harmonic oscillator, Helv. Phys. Acta 46 (1973), 191-200.

[126] A. F. Nikiforov, S. K. Suslov, and V. B. Uvarov, Classical Orthogonal Polynomials of a Discrete Variable, Springer-Verlag, Berlin, New York, 1991.

[127] A. Ourjoumtsev, A. Kubanek, M. Koch, C. Sames, P. W. H. Pinkse, G. Rempe, and K. Murr, Observation of squeezed light from one atom excited with two photons, Nature 474 (2011), 623-626.

[128] W. Paul, Electromagnetic traps for charged and neutral particles, Rev. Mod. Phys. 62 (1990) \# 3, 531-540.

[129] A. M. Perelomov, Generalized Coherent States and Their Applications, Springer-Verlag, Berlin, 1986.

[130] I. Pikovski, M. R. Vanner, M. Aspelmeyer, M. S. Kim, and Č. Brukner, Probing Planck-scale physics with quantum optics, Nature Physics 8 (2012), 393-397.

[131] J. Ries, B. Brezger, and A. I. Lvovsky, Experimental vacuum squeezing in rubidium vapor via self-rotation, Phys. Rev. A 68 (2003), 025801 (4 pages).

[132] G. Rempe, F. Schmidt-Kaler, and H. Walther, Observation of sub-Poissonian photon statistics in a micromaser, Phys. Rev. Lett. 64 (1990) \# 23, 2783-2786.

[133] G. Rempe, H. Walther, and N. Klein, Observation of quantum collapse and revival in a one-atom maser, Phys. Rev. Lett. 58 (1987) \# 4, 353-356.

[134] S. Rosencrans, Perturbation algebra of an elliptic operator, J. Math. Anal. Appl. 56 (1976) \# 2, 317-329.

[135] Ch. Roos, Th. Zeiger, H. Rohde, H. C. Nägerl, J. Eschner, D. Leibfried, F. Schmidt-Kaler, and R. Blatt, Quantum state engineering on an optical transition and decoherence in a Paul trap, Phys. Rev. Lett. 83 (1999) \# 23, 4713-4716.

[136] D. J. Rowe, The two-photon laser beam as a breathing mode of the electromagnetic field, Can. J. Phys. 56 (1978), 442-446. 
[137] B. Sanborn, S. K. Suslov, and L. Vinet, Dynamic invariants and Berry's phase for generalized driven harmonic oscillators, Journal of Russian Laser Research 32 (2011) \# 5, 486-494; see also arXiv:1108.5144v1 [math-ph] 25 Aug 2011.

[138] M. F. Santos, G. Giedke, and E. Solano, Noise-free measurement of marmonic oscillators with instantaneous interactions, Phys. Rev. Lett. 98 (2007), 020401 (4 pages).

[139] L. I. Schiff, Quantum Mechanics, third edition, McGraw-Hill, New York, 1968.

[140] S. Schiller, G. Breitenbach, S. F. Pereira, T. Müller, and J. Mlynek, Quantum statistics of the squeezed vacuum by measurement of the density matrix in the number state representation, Phys. Rev. Lett. 77 (1996) \# 14, 2933-2936.

[141] W. P. Schleich, Quantum Optics in Phase Space, Wiley-Vch Publishing Company, Berlin etc, 2001.

[142] G. Schrade, V. I. Man'ko, W. P. Schleich, and R. J. Glauber, Wigner functions in the Paul trap, Quantum Semiclass. Opt. B 7 (1995) \# 3, 307-325.

[143] E. Schrödinger, Quantisierung als Eigenwertproblem II, Annalen der Physik, 79 (1926), 489-527; see also Collected Papers on Wave Mechnics, Blackie \& Son Ltd, London and Glascow, 1928, pp. 13-40, for English translation of Schrödinger's original paper.

[144] E. Schrödinger, Der stetige Übergang von der Mikro-zur Makro Mechanik, Die Naturwissenshaften, 28 (1926), 664-666; see also Collected Papers on Wave Mechnics, Blackie \& Son Ltd, London and Glascow, 1928, pp. 4144, for English translation of Schrödinger's original paper.

[145] R. Schützhold, G. Plunien, and G. Soff, Trembling cavities in the canonical approach, Phys. Rev. A 57 (1998) \# 4, 2311-2318.

[146] E. Shchukin, Th. Kiesel, and W. Vogel, Generalized minimum-uncertainty squeezed states, Phys. Rev. A 79 (2009), 043831 (7 pages).

[147] B. W. Shore and P. L. Knight, The Jaynes-Cummings model, J. Mod. Opt. 40 (1993) \# 7, $1195-1238$.

[148] J. C. Slater, Microvawe Electronics, D. van Nostrand Co. Inc., New York, chapter 4, 1950.

[149] R. E. Slusher, L. W. Hollberg, B. Yurke, J. C. Mertz, and J. F. Valleys, Observation of squeezed states generated by four-wave mixing in an optical cavity, Phys. Rev. Lett. 55 (1985) \# 22, 2409-2412.

[150] S. Stenholm, Amplification of squeezed states, Optics Communications 58 (1986) \# 3, 177-180.

[151] D. Stoler, Equivalence Classes of Minimum Uncertainty Packets, Phys. Rev. D 1 (1970) \# 12, 3217-3219.

[152] D. Stoler, Equivalence Classes of Minimum Uncertainty Packets. II, Phys. Rev. D 4 (1971) \# 6, $1925-1926$.

[153] E. Suazo and S. K. Suslov, Soliton-like solutions for nonlinear Schrödinger equation with variable quadratic Hamiltonians, Journal of Russian Laser Research 33 (2012) \# 1, 63-82; arXiv:1010.2504v4 [math-ph] 24 Nov 2010 .

[154] E. Suazo, S. K. Suslov, and J. M. Vega-Guzmán, The Riccati equation and a diffusion-type equation, New York J. Math. 17a (2011), 225-244.

[155] E. Suazo, S. K. Suslov, and J. M. Vega-Guzmán, The Riccati system and a diffusion-type equation, arXiv: 1102.4630v1 [math-ph] 22 Feb 2011.

[156] S. K. Suslov, Dynamical invariants for variable quadratic Hamiltonians, Physica Scripta 81 (2010) \# 5, 055006 (11 pp); see also arXiv:1002.0144v6 [math-ph] 11 Mar 2010.

[157] S. K. Suslov, On integrability of nonautonomous nonlinear Schrödinger equations, Proc. Amer. Math. Soc. 140 (2012) \# 9, 3067-3082; see also arXiv:1012.3661v3 [math-ph] 16 Apr 2011.

[158] S. K. Suslov, An analog of the Berry phase for simple harmonic oscillators, Physica Scripta 87 (2013) \# 3, 038118 (4 pages); arXiv:1112.2418v1 [quant-ph] 12 Dec 2011.

[159] S. K. Suslov, http://hahn.la.asu.edu/〜suslov/curres/index.htm; see Mathematica notebook: BerrySummary.nb.

[160] T. Takabayasi, The formulation of quantum mechanics in terms of ensemble in phase space, Prog. Theor. Phys. 11 (1954) \# 4, 341-373.

[161] W. G. Unruh, Notes on black-hole evaporation, Phys. Rev. D 14 (1976) \# 4, 870-892.

[162] H. Vahlbruch, M. Mehmet, S. Chelkowski et al, Observation of squeezed light with 10-dB quantum-noise reduction, Phys. Rev. Lett. 100 (2008), 033602 (4 pages).

[163] P. Verkerk, B. Lounis, C. Salomon, and C. Cohen-Tannoudji, Dynamics and spatial order of cold Cesium atoms in a periodic optical potential, Phys. Rev. Lett. 68 (1992) \# 26, 3861-3864.

[164] M. B. Vinogradova, O. V. Rudenko, and A. P. Sukhorukov, Theory of Waves, Nauka, Moscow, 1979 [in Russian]. 
[165] W. Vogel and R. L. de Matos Filho, Nonlinear Jaynes-Cummings dynamics of a trapped ion, Phys. Rev. A 52 (1995) \# 5, 4214-4217.

[166] V. I. Vysotskii, M. V. Vysotskyy, and S. V. Adamenko, Formation and application of correlated states in nonstationary systems at low energies of interacting particles, J. Exp. Theor. Phys.-JETP 114 (2012) \# 2, $243-252$.

[167] D. F. Walls, Squeezed states of light, Nature 306 (1983) November 10, 141-146.

[168] D. F. Walls and G. J. Milburn, Quantum Optics, Springer, Berlin, Heidelberg, 2008.

[169] S. Weinberg, The Quantum Theory of Fields, volumes 1-3, Cambridge University Press, Cambridge, 1998.

[170] S. Weinberg, Lectures on Quantum Mechanics, Cambridge University Press, New York, 2013.

[171] E. Wigner, On the quantum correction for thermodynamic equilibrium, Phys. Rev. 40 (1932), 749-759.

[172] C. M. Wilson, G. Johansson, A. Pourkabirian, M. Simoen, J. R. Johansson, T. Duty, F. Nori, and P. Delsing, Observation of the dynamical Casimir effect in a superconducting circuit, Nature 479 (2011) November 17, 376-379.

[173] J. Q. You and F. Nori, Atomic physics and quantum optics using superconducting circuits, Nature 474 (2011), 589-597.

[174] H. P. Yuen, Two-photon coherent states of the radiation field, Phys. Rev. A 13 (1976) \# 6, 2226-2243.

Mathematical, Computational and Modeling Sciences Center, Arizona State University, Tempe, AZ 85287-1904, U.S.A.

E-mail address: sergeykryuchkov@yahoo.com

School of Mathematical and Statistical Sciences, Arizona State University, Tempe, AZ 852871804, U.S.A.

E-mail address: sks@asu.edu

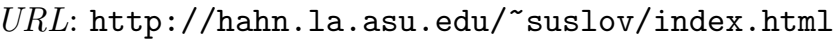

Mathematical, Computational and Modeling Sciences Center, Arizona State University, Tempe, AZ 85287-1904, U.S.A.

E-mail address: jmvega@asu.edu 